\title{
Pearls and pitfalls of imaging features of pancreatic cystic lesions: a case-based approach with imaging-pathologic correlation
}

\author{
Kumi Ozaki ${ }^{1}$ (]) Hiroshi lkeno ${ }^{2} \cdot$ Yasuharu Kaizaki $^{3} \cdot$ Kazuya Maeda $^{4} \cdot$ Shohei Higuchi $^{5} \cdot$ Nobuyuki Kosaka $^{1}$. \\ Hirohiko Kimura ${ }^{1} \cdot$ Toshifumi Gabata $^{2}$
}

Received: 14 July 2020 / Accepted: 11 August 2020 / Published online: 25 August 2020

(c) Japan Radiological Society 2020

\begin{abstract}
A variety of neoplastic and non-neoplastic lesions of the pancreas can present with a predominantly cystic architecture. These lesions are increasingly being detected as incidental findings on routine cross-sectional imaging following technological advances in these techniques and their widespread use. The different histopathological behaviors show various common and uncommon imaging findings, and some cases show similar appearance in spite of different histopathology. Each lesion requires specific management because of the differing risk of progression to malignancy, and an accurate imaging diagnosis is crucial. The typical imaging characteristics that differentiate pancreatic cystic lesions have been well described and fully summarized. However, in addition to a small percentage of cases that shows uncommon imaging findings, a substantial percentage of cystic lesions shows overlapping imaging findings that can lead to radiological misdiagnosis. For appropriate diagnosis and optimal treatment strategy, it is important to know the uncommon and overlapping imaging findings of these lesions, in addition to familiarity with the typical aspects. In this article, we reconfirm the well-known characteristic imaging features of pancreatic cystic lesions and present several diagnostically challenging cases, focusing on the uncommon and overlapping imaging findings.
\end{abstract}

Keywords Pseudocyst $\cdot$ Serous cystadenoma $\cdot$ Intraductal papillary mucinous neoplasm $\cdot$ Mucinous cystic neoplasm $\cdot$ Solid pseudopapillary neoplasm

\section{Introduction}

Pancreatic cystic lesions are being detected with increasing frequency because of the widespread use and improved quality of cross-sectional imaging techniques [1-3]. A cystic lesion can be present in a wide variety of pancreatic

\section{Kumi Ozaki}

ozakik-rad@umin.org

1 Department of Radiology, Faculty of Medical Sciences, University of Fukui, 23-3 Matsuoka-Shimoaizuki, Eiheiji, Fukui 910-1193, Japan

2 Department of Radiology, Kanazawa University Graduate School of Medical Sciences, Kanazawa, Japan

3 Department of Diagnostic Pathology, Fukui Prefectural Hospital, Fukui, Japan

4 Department of Surgery, Fukui Prefectural Hospital, Fukui, Japan

5 Department of Pathology, Faculty of Medical Sciences, University of Fukui, Fukui, Japan conditions, including cystic neoplasms, non-neoplastic lesions, and solid tumors with cystic degeneration. Furthermore, several peripancreatic cystic lesions should be considered in the differential diagnosis. An accurate imaging diagnosis is crucial because appropriate management, such as surgery, conservative following, or further examination, such as endoscopic ultrasound (EUS) with or without fineneedle aspiration, differs depending on the lesion type $[4,5]$.

The mainstay of assessment of pancreatic cystic lesions is cross-sectional imaging: computed tomography (CT) and magnetic resonance imaging (MRI), including MR cholangiopancreatography, which can provide objective and reproducible information. These modalities can be used independently or in combination to assist in the diagnosis and management of pancreatic cystic lesions. Compared with MRI, CT has superior spatial resolution and has the additional advantage of depicting calcifications [6]. MRI has superior contrast resolution and has advantages over CT with respect to depicting characterizing features, such as morphology, internal septation, mural nodules, 
fluid content, and the relationship between pancreatic cystic lesions and the pancreatic duct [7].

Typical imaging characteristics for differentiating between pancreatic cystic lesions have been well described and fully summarized in an array of literature and presentations [7-13]. In the clinical setting, however, we encounter challenging cases not infrequently in preoperative imaging diagnosis, for the reason that in addition to the small percentage of cases showing uncommon imaging findings, a substantial percentage of cystic lesions shows overlapping imaging findings that can lead to radiological misdiagnosis. We should be familiar not only with the typical aspects of these lesions, but also the uncommon and overlapping imaging findings, to enable the correct diagnosis and optimal treatment strategy.

In this article, we reconfirm the well-known characteristic imaging features of pancreatic cystic lesions and present various challenging diagnostic cases, focusing on the uncommon and overlapping imaging findings.

\section{Classification of pancreatic cystic lesions}

Cystic pancreatic lesions constitute a diverse category that includes inflammatory lesions as well as neoplasms ranging from benign lesions and low-grade indolent neoplasia, to frankly malignant tumors. They are divided into three categories: (1) primary neoplastic cysts, which include serous cystadenoma (Figs. 1, 2, 3, 4), mucinous cystic neoplasm (MCN; Fig. 5), intraductal papillary mucinous neoplasm (IPMN; Figs. 6, 7, 8, 9), and solid pseudopapillary neoplasm (SPN; Fig. 10); (2) non-neoplastic cysts, which include pseudocyst (Figs. 11, 12, 13), congenital cyst, retention cyst, hydatid cyst, lymphoepithelial cyst (Fig. 14), and epidermoid cyst of intrapancreatic accessory spleen (Fig. 15); and (3) various solid neoplasms undergoing cystic changes including neuroendocrine tumor (Figs. 16, 17, 18), metastatic tumor, ductal adenocarcinoma, and acinar cell cystadenocarcinoma. In the actual clinical setting, the latter two tumors rarely show complete cystic configurations that mimic real pancreatic cystic lesions. Furthermore, several peripancreatic cystic lesions should be considered in the differential diagnosis; these include

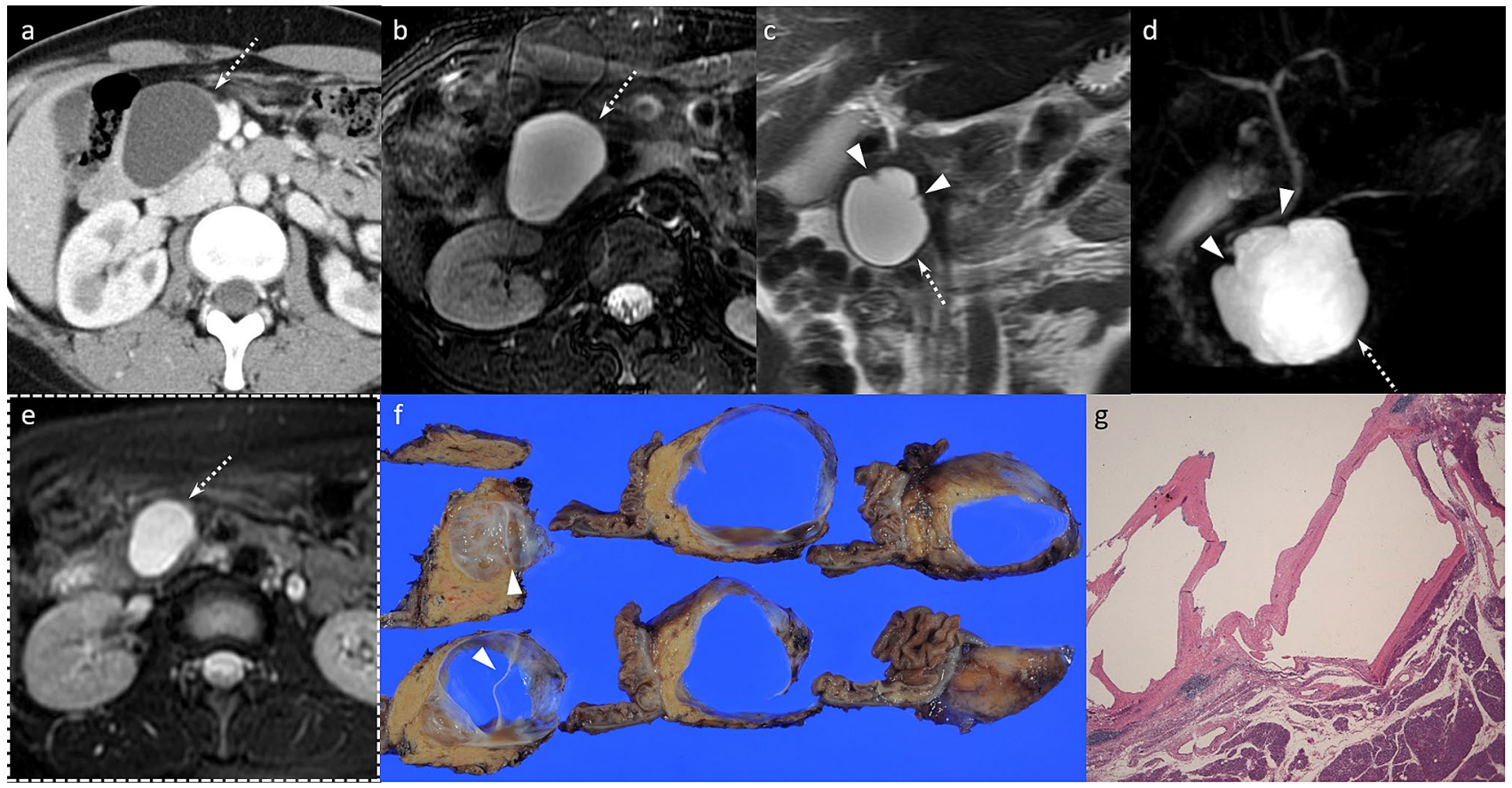

Fig. 1 A 29-year-old woman with an enlarging unilocular cystic lesion in the pancreatic head mimicking mucinous cystic neoplasm that was diagnosed surgically as macrocystic serous cystadenoma. Serous cystadenoma sometimes shows enlargement and lobulated contours should be noted. a Portal phase CT shows a unilocular and relatively large cystic lesion (approximately $6 \mathrm{~cm}$ in diameter) in the pancreatic head (arrow). b The cystic lesion shows obvious and homogeneous hyperintensity (arrow) on T2-weighted fat-suppressed MRI. c Coronal T2-weighted MRI shows the unilocular cystic lesion (arrow) with lobulated contours (arrowheads). d The unilocular cystic lesion (arrow) with lobulated contours (arrowheads) is visualized on MR cholangiopancreatography. e The lesion was smaller in size (approximately $2.5 \mathrm{~cm}$ in diameter, arrow) on T2-weighted fatsuppressed MR obtained 3 years previously. $\mathbf{f}$ The resected specimen shows a thin-walled cystic mass with septation (arrowheads). $g$ The epithelium of the cyst consists of simple cuboidal epithelial cells with clear cytoplasm [hematoxylin and eosin (HE) staining; original magnification $\times 10$ ] 


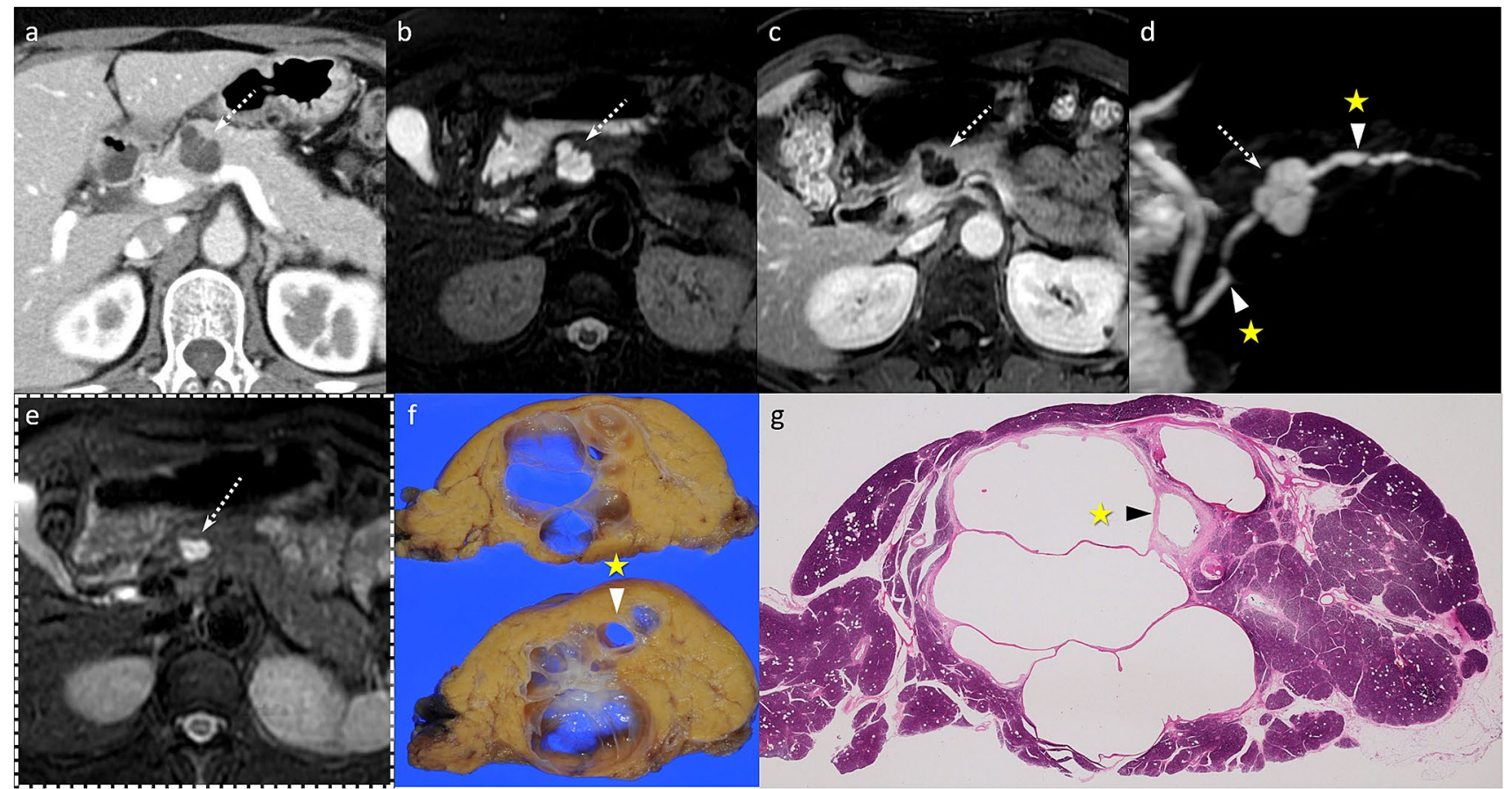

Fig. 2 A 52-year-old woman with an enlarging multilocular cystic lesion in the pancreatic head accompanied by dilated main pancreatic duct (MPD), mimicking branch duct or mixed intraductal papillary mucinous neoplasm (IPMN). The lesion was diagnosed surgically as macrocystic serous cystadenoma. Serous cystadenoma sometimes shows enlargement and/or dilated MPD due to compression. a Arterial phase CT shows an approximately $3 \mathrm{~cm}$ diameter, multilocular cystic lesion in the pancreatic head (arrow). b T2-weighted fat-suppressed MRI also shows the multilocular cystic nature of the lesion (arrow). c The lesion shows no enhancement on the delayed phase of dynamic contrasted MRI (arrow). d The cystic lesion is clearly depicted on MR cholangiopancreatography (arrow). The MPD is

neurogenic tumor (Fig. 19), metastatic lymph node (Fig. 19), walled-off necrosis (after pancreatitis; Fig. 19), and others (Fig. 20) (Table 1).

On the basis of involvement of the pancreatic duct, IPMNs are classified as branch duct IPMN, main duct IPMN, or mixed variant IPMN involving both the branch and main pancreatic ducts [14-16]. Main duct IPMNs rarely completely mimic pancreatic cystic lesions, but branch duct and mixed IPMNs can have a similar appearance to other benign cystic lesions. All morphologic variants of IPMN can progress to cancer [17]. MCNs and SPNs have also malignant potential, and should be discriminated from benign lesions.

\section{Clinical information}

Pancreatic cystic lesions not only have diverse histologic and imaging appearances, but also differ in their clinical presentation, including the frequency, age and gender of the dilated (arrowheads with stars) both upstream and downstream of the lesion, presumably due to compression and age-related change, respectively. No communication is observed between the cystic lesion and the MPD. e The lesion was smaller in size (approximately $1.3 \mathrm{~cm}$ in diameter, arrow) on T2-weighted fat-suppressed MRI acquired 4 years previously. $f$ The resected specimen shows a thin-walled lesion comprising several macro-cysts with septa. A dilated MPD is also observed (arrowhead). No communication is observed between the cystic lesion and the MPD. $\mathbf{g}$ The walls of the cyst consist of simple cuboidal epithelial cells with clear cytoplasm (HE staining; loupe image). A dilated MPD is also observed (arrowhead)

patient, and clinical symptoms (Table 2). These clinical data can help to narrow the differential diagnosis and stratify the risk of malignancy.

\section{Frequency}

The frequency of the lesion type should be considered in the differential diagnosis because clinically, it is more likely that doctors will encounter an atypical presentation of a common tumor than a rare cystic lesion. The most common non-neoplastic pancreatic cystic lesions are pseudocysts, which comprise approximately one-third of all pancreatic cystic lesions [18]. The most common neoplastic lesions are IPMN, serous cystadenoma, and MCN $[1,15,19]$. According to histopathological data, the frequency of pancreatitisassociated pseudocyst has been reported as $34 \%$, followed by IPMN (24\%), serous cystadenoma (10\%), MCN (8\%), and SPN (3\%) [20], although these data were collected from various sources, including from surgery, imaging, autopsy, and histopathological data. Furthermore, another 


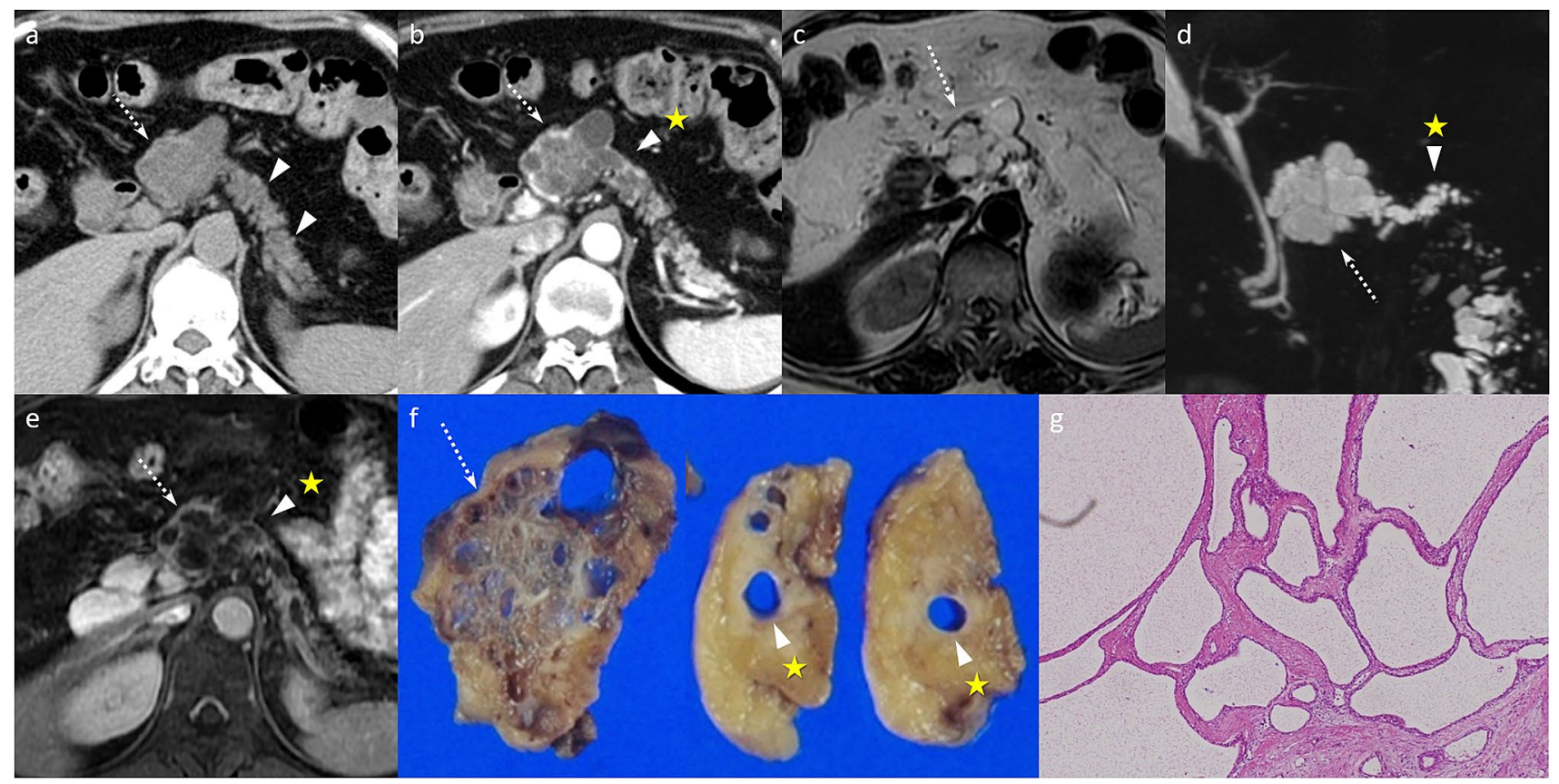

Fig. 3 A 73-year-old woman with a cystic lesion accompanied by dilated MPD, mimicking mixed IPMN that was diagnosed surgically as macrocystic serous cystadenoma. Serous cystadenoma is sometimes accompanied by dilated MPD due to compression. a Unenhanced CT reveals an approximately 4-cm-diameter, multilocular cystic lesion (arrow) in the pancreatic head. Mild parenchymal atrophy without calcification is seen in the body and tail of the pancreas (arrowheads). b Arterial phase CT shows the multilocular cystic lesion (arrow) accompanied by dilated MPD (arrowhead). c T2-weighted MRI also shows the lesion (arrow). d The cystic

study showed that these neoplastic and non-neoplastic cystic lesions account for $90 \%$ of all pancreatic cystic lesions [21]. Other cystic pancreatic lesions account for fewer than $10 \%$ of cases, and include uncommon pathologic findings, such as lymphoepithelial cyst, epidermoid cyst of intrapancreatic accessory spleen, ductal adenocarcinoma or neuroendocrine neoplasm with cystic degeneration, and cystic degeneration in other solid pancreatic neoplasms $[4,22]$.

In addition, neuroendocrine neoplasm is relatively rare and accounts for only 1-2\% of pancreatic tumors [23], and extensive cystic change is seen in approximately $5-10 \%$ of tumors [24]. Intrapancreatic accessory spleen is very rare and cyst formation even more rare [25, 26].

\section{Age and sex}

Some pancreatic cystic lesions are particularly related to age and sex. MCN is common among middle-aged women [27], in other words, rarely developing in males. SPN occurs predominantly in young women (median age, 30 years) [28]. Although not a determinable factor in the differential diagnosis, age and gender predominance must also be considered lesion is clearly depicted on MR cholangiopancreatography (arrow). Dilated MPD in the body and tail is also depicted (arrowhead). e Delayed phase of dynamic contrasted MRI shows no wall thickening or enhanced mural nodules in the lesion (arrow), and also depicts the dilated MPD (arrowhead). There appears to be communication between the cystic lesion and the MPD. f The resected specimen shows a thin-walled multilocular cystic mass with septations (arrow) and a dilated MPD (arrowheads). $\mathbf{g}$ The walls of the cyst consist of simple cuboidal epithelial cells with clear cytoplasm (HE staining; original magnification $\times 20$ )

for serous cystadenoma and IPMN. Serous cystadenoma occurs most frequently in older women (median age, 65 years) [29]; whereas IPMN shows a small male gender predisposition $[31,32]$ but occurs most frequently in older men (median age, 65 years) [30]. Note that the risk of both IPMN development and malignant degeneration increases with age [31-33]. IPMN diagnosed in older patients is more likely to progress to high-risk stigmata despite the fact that cancer-related death is not affected by age [34].

Neuroendocrine tumors occur most frequently in those aged over 50 years, with a slight male predominance [24]. Pancreatic ductal adenocarcinoma predominantly affects the elderly population, with $80 \%$ of diagnosed patients aged more than 60 years, median age at diagnosis of 72 years, and a slight male predominance [35]. In contrast, pseudocyst can occur at any age and in either sex [36].

\section{Symptoms}

A large number of pancreatic cystic lesions are detected incidentally during the imaging work-up for an unrelated medical problem [1-3]. In other words, most pancreatic 


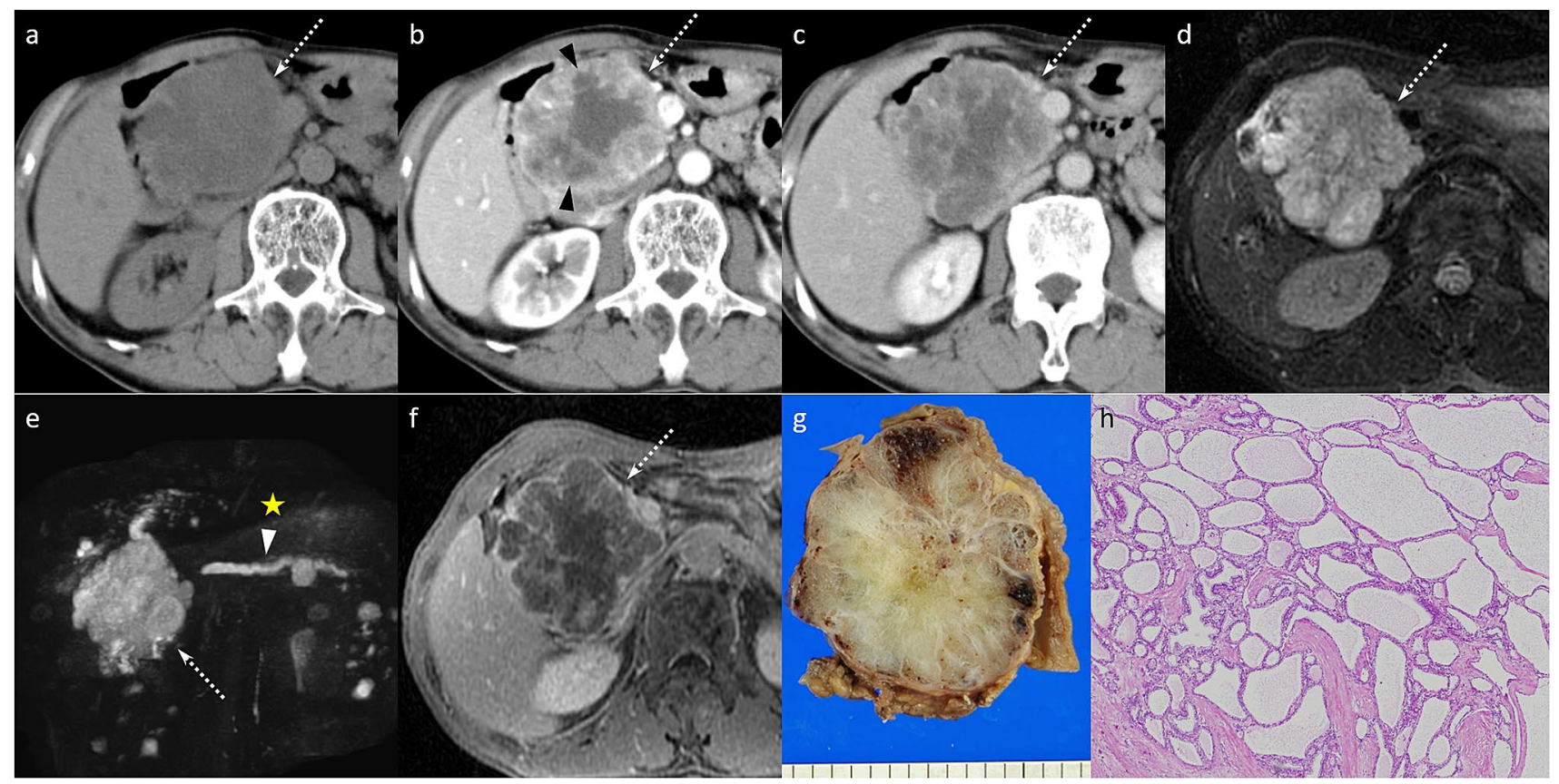

Fig. 4 A 72-year-old man with a huge mass of the pancreatic head, mimicking a solid mass with cystic degeneration, that was diagnosed surgically as serous cystadenoma. Mixed micro- and macrocystic serous cystadenoma shows inhomogeneous early staining, and enhancement of the fibrous septa may mimic a solid mass accompanied by degeneration. Note that the MR cholangiopancreatographic image characterizes the cystic nature of the lesion and the lobulated contours. a Unenhanced CT shows a huge low-density mass of the pancreatic head (arrow) without calcification, approximately $7 \mathrm{~cm}$ in diameter. b Arterial phase CT reveals inhomogeneous early enhancement (arrow) and a non-enhancing area (arrowheads), having the appearance of cystic degeneration of a solid mass. c Equilibrium

cystic neoplasms are asymptomatic. Note, however, that MCN can be symptomatic in up to $10 \%$ of cases (Fig. 5) [37]. When present, symptoms are caused by the mass effect of these often large neoplasms.

In most cases, IPMN is not accompanied by acute symptoms [38]. Due to transient mucinous ductal obstruction, however, both branch and main duct IPMNs can cause mild and often recurrent flares of acute pancreatitis in 7-34.6\% of cases [39-42]. In addition, obstructive jaundice in a patient with IPMN in the pancreatic head may suggest the features of high-risk malignant lesions, especially in cases accompanied by other high-risk malignant features including mass size $>3 \mathrm{~cm}$, enhancing solid component, and main pancreatic duct (MPD) size $\geq 10 \mathrm{~mm}$.

Approximately $60-90 \%$ of neuroendocrine tumors are nonfunctioning and are detected as an incidental finding on imaging [24, 43, 44], whereas functioning neuroendocrine tumors hypersecrete single or multiple hormones, causing a constellation of symptoms and often a dramatic presentation [45]. phase CT shows inhomogeneous enhancement (arrow) and a nonenhanced area (arrowhead), suggesting cystic degeneration. d The mass shows obvious hyperintensity on $\mathrm{T} 2$-weighted fat-suppressed MRI. e MR cholangiopancreatography clearly depicts the cystic mass with lobulated contours (arrow), and a mildly dilated MPD (arrowhead). f Delayed phase image of dynamic contrasted MRI depicts the wall and septations of the lesion (arrow). $\mathbf{g}$ The resected specimen shows a pathognomonic sponge-like configuration consisting of innumerable micro- and macro-cysts. $\mathbf{h}$ The cyst walls consist of simple cuboidal epithelial cells with clear cytoplasm (HE staining; original magnification $\times 20$ )

Although a complete cystic appearance is rare in ductal adenocarcinoma, patients can present with symptoms, such as abdominal pain or discomfort, weight loss, and jaundice or recurrent pancreatitis, that indicate that the lesion is either in communication with the pancreatic ductal system or obstructing the pancreatic or biliary duct [46]. Pseudocysts typically occur with acute pancreatitis or may develop insidiously in the setting of chronic pancreatitis [47].

\section{Imaging key points in differential diagnosis}

Radiological imaging plays a crucial role in the differential diagnosis. Technological innovations in CT and MRI are particularly valuable in delineating the key imaging features of cystic lesions, changes in the pancreatic parenchyma and pancreatic duct, and peripancreatic findings, described as follows (Table 3 ). The common and typical imaging features of pancreatic cystic lesions are summarized in Table 4. 


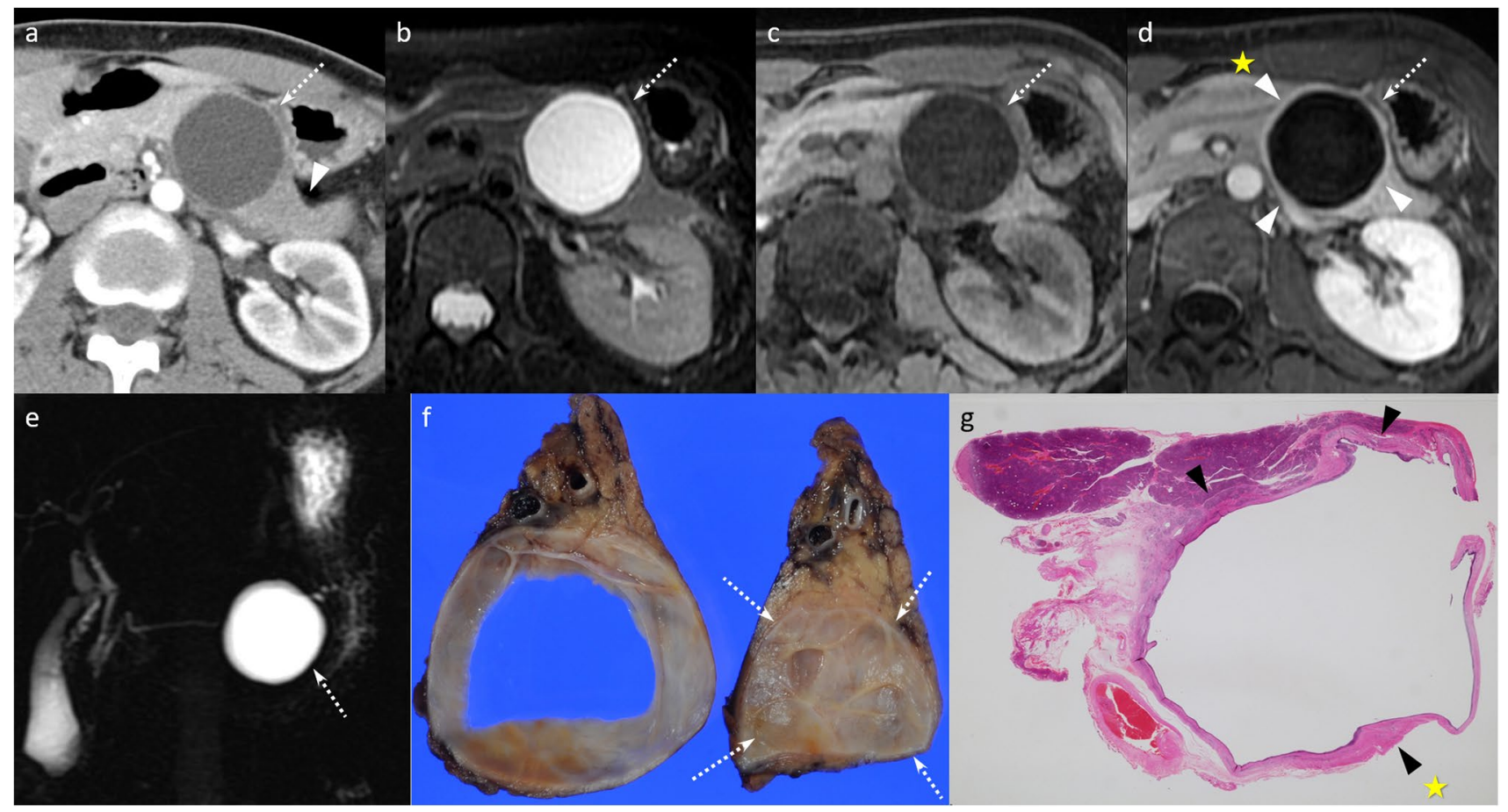

Fig. 5 A 60-year-old woman with a unilocular cystic lesion mimicking macrocystic-type serous cystadenoma that was diagnosed surgically as mucinous cystic neoplasm. The homogeneous wall thickening is noteworthy. a Arterial phase CT reveals a unilocular cystic lesion (arrow) without calcification or mural nodules. The parenchyma of the tail shows slightly poorer enhancement (arrowhead), suggesting acute pancreatitis. b T2-weighted fat-suppressed MRI shows a unilocular cystic lesion without septation or lobulated contours (arrow). The contents show clear homogeneous hyperintensity.

\section{Morphologic types of cystic lesions of the pancreas}

The morphological characteristics are the most important and useful in the differential diagnosis, and can be classified based on specific CT and MR imaging features into four categories: unilocular, microcystic, macrocystic, and cysts with solid components $[8,9,48]$, although there is unignorable overlap among the imaging findings.

Unilocular cysts are thin-walled simple cystic lesions without solid components or internal septa $[8,48]$. Pseudocyst is the most common lesion in this category $[8,48]$. IPMN and MCN are also common, whereas uncommon lesions include serous cystadenoma (oligocystic variant $<10 \%$; Fig. 1), lymphoepithelial cyst (Fig. 14), epidermoid cyst (Fig. 15), and neuroendocrine tumors with cystic degeneration (Figs. 17, 18) [8, 49]. Neuroendocrine neoplasm, especially if small (Figs. 17, 18), can be exclusively cystic, making the differential diagnosis with cystic neoplasms, unilocular serous cystadenomas (Fig. 1) or retention cysts extremely difficult.

Microcystic lesions typically present with multiple tiny cysts (more than six, each measuring $<2 \mathrm{~cm}$ ) with lobulated c Precontrast T1-weighted fat-suppressed MRI shows the unilocular cystic lesion with no mural nodules or septa (arrow). The content shows clear hypointensity. d Delayed phase image of dynamic contrasted MRI depicts homogeneous wall thickening (arrowheads) of the cystic lesion (arrow). No mural nodules are observed. e MR cholangiopancreatography clearly depicts the unilocular cystic lesion (arrow). f The resected specimen shows a cystic mass with septations (arrows). $\mathbf{g}$ Loupe image shows the cyst wall lined by tall columnar cells without atypia (HE staining)

contours $[29,49]$. This category includes most typical serous cystadenomas (polycystic or honeycomb type, comprising almost $70 \%$ and $20 \%$ of serous cystadenomas, respectively). The numerous tiny cysts show avid enhancement on the arterial phase images owing to the presence of a vascular epithelial lining despite their completely cystic nature (Fig. 4). This appearance can masquerade as a solid pancreatic neoplasm, such as neuroendocrine tumor (Fig. 16) or a metastatic lesion from a primary cancer, such as renal cell carcinoma or melanoma. Delayed phase contrast-enhanced images can reveal the microcysts and the enhancing stroma. T2-weighted MRI and MR cholangiopancreatography can confirm the presence of high-signal-intensity microcysts [29, 49]; nevertheless, the differential diagnosis between serous cystadenoma and neuroendocrine tumor can be challenging (Figs. 4, 16).

Macrocystic lesions contain fewer cysts than microcystic lesions, and the cysts are often $>2 \mathrm{~cm}$ in diameter $[8,15,22,27,29]$. MCN and branch duct IPMN are mainly included in this category, as well as uncommon lesions, such as serous cystadenoma (oligocystic variant), lymphoepithelial cyst, epidermoid cyst, and 


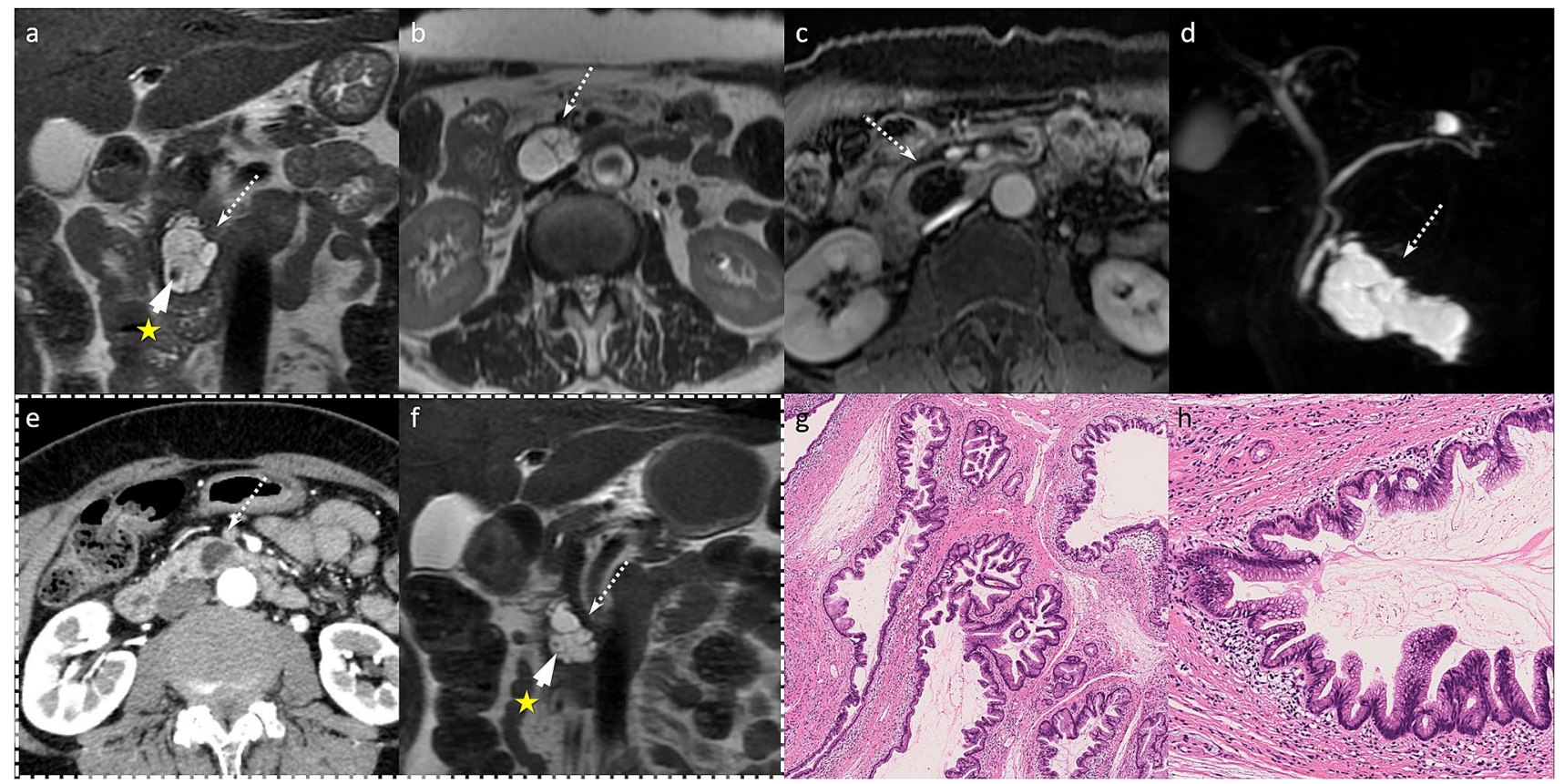

Fig. 6 A 76-year-old woman with a cystic lesion in the pancreatic head mimicking enlarging branch duct IPMN and a mural nodulelike lesion suggestive of a "worrisome feature", which was diagnosed surgically as branch duct IPMN with mucin plugs, without malignancy. Branch duct IPMN with low-grade dysplasia often contains mucin plugs, which show hypointensity on T2-weighted MRI without enhancement, and should be distinguished from enhancing mural nodules suggestive of high-risk stigmata and worrisome features. a T2-weighted coronal MRI shows an approximately $4.5-\mathrm{cm}-$ diameter $(>3 \mathrm{~cm})$, enlarging, multilocular cystic lesion in the pancreatic head (arrow) with a hypointense nodular lesion (arrowhead). b T2-weighted axial MRI shows the cystic lesion (arrow). c Delayed phase image of dynamic contrasted MRI shows no enhancement of the cystic lesion (arrow). d Multilocular cystic lesion (arrow) of the pancreatic head has the appearance of a branch duct IPMN on MR cholangiopancreatography. e The cystic lesion was smaller in size (approximately $1.3 \mathrm{~cm}$ in diameter, arrow) on portal phase CT obtained 2.5 years previously. $\mathbf{f}$ The lesion (arrow) and the mural nodule-like lesion (arrowhead) were smaller in size on T2-weighted coronal MRI acquired 1.5 years previously. $\mathbf{g}$, $\mathbf{h}$ Histopathological images reveal mucin-producing epithelial cells without nuclear atypia forming a papillary arrangement, which was diagnosed as IPMN with lowgrade dysplasia [HE staining; original magnification $\times 10($ g $), \times 20$ (h)] neuroendocrine tumor, with cystic degeneration. More specifically, serous cystadenoma tends to show cyst-oncyst formation and lobulated contours, MCN shows septa with cyst-in-cyst formation and oval shape, and branch duct IPMN shows grape-like agglomerations with cystby-cyst formation. Furthermore, communication between cysts and side branches or the MPD may be displayed in IPMN, whereas neither serous cystadenomas nor MCN communicate with the pancreatic duct [50]. Despite these characteristics, differential diagnosis among serous cystadenoma (Figs. 2, 3), branch duct IPMN, and MCN can be challenging.

Cysts with solid components include true cystic tumors with a solid portion, such as MCN and IPMN, suspicious of malignancy; and solid tumor with cystic degeneration, such as SPN, neuroendocrine tumor, ductal adenocarcinoma, and metastatic lesions [8]. In addition, lymphoepithelial cyst or epidermoid cyst in intrapancreatic spleen may show a similar solid component.

\section{Wall}

Findings of the wall of cystic lesions are another important element in differential diagnosis. The key features are thickening, enhancement, and wall irregularity. These can be observed in MCN, IPMN, SPN, pseudocyst, lymphoepithelial cyst, and epidermoid cyst, and do not necessarily suggest malignancy, depending on the histopathology.

The typical wall of an MCN is homogeneously thick and shows enhancement in the delayed phase of multiphasic contrasted imaging, which correlates with the fibrotic changes seen in histologic analysis (Fig. 5) [37, 51]; these wall characteristics do not always indicate frank malignancy. In IPMN, thickening, enhancing or irregularity of the wall are indicative features of malignancy, in addition to size $>3 \mathrm{~cm}$ [16]. In other words, indistinct wall thickening alone does not indicate malignancy (Fig. 8). Thickened wall is common in SPN but does not necessarily indicate malignant transformation, although SPN does have malignant potential 


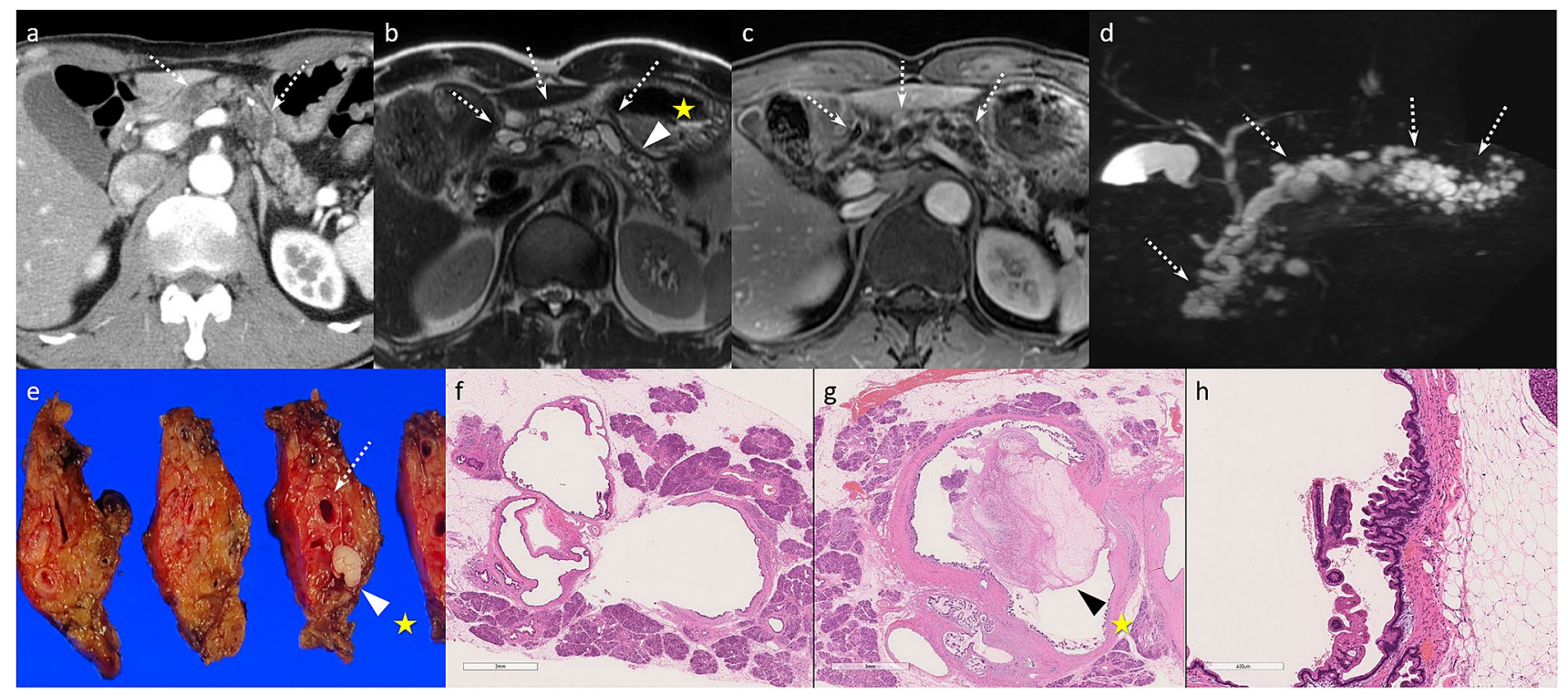

Fig. 7 A 66-year-old man with dilated main and branch pancreatic ducts with mural nodule mimicking mixed IPMN with malignancy, which was diagnosed surgically as mixed IPMN without malignancy. IPMNs often contain mucin plugs, which should be discriminated from enhanced mural nodules suggesting malignancy. a Arterial phase CT depicts dilated main and branch pancreatic ducts (arrows). b T2-weighted MRI shows the dilated pancreatic ducts (arrows) and a hypointense mural nodule (arrowhead). c Coronal image of the portal phase of dynamic contrasted MRI shows the dilated pancreatic ducts

$[28,52]$. On the other hand, vascular invasion, lymph node involvement, and deep invasion of surrounding tissues may be suggestive of malignant transformation [53].

Neuroendocrine neoplasm with cystic change can show partial or entire wall thickening when the tumor undergoes complete cystic change (Figs. 17, 18).

Pseudocyst can show thickening of the wall due to inflammation or hemorrhage (Figs. 11, 12).

Lymphoepithelial or epidermoid cysts are lined by squamous epithelium and surrounded by dense lymphoid tissue and may show wall enhancement or thickening [25, 26, 54, 55].

\section{Mural nodules or solid component and mimicking}

Mural nodules or solid enhancing components are typical features and high-risk markers of mucinous cystic neoplasms including MCN and IPMN, and these should be interpreted carefully. In some cases, it is difficult to distinguish mural nodules from mucous plugs, which are relatively small nodular lesions that do not enhance but can have a similar appearance to mural nodules (Figs. 6, 7) [56-58]. Due to its high spatial resolution, EUS enables mucin plugs to be reliably distinguished from true mural nodules, with sensitivity and specificity of $89-96 \%$ (arrows). The mural nodule depicted in b shows no enhancement. d MR cholangiopancreatography depicts the dilated pancreatic ducts (arrows). e The resected specimen shows dilated main and branch pancreatic ducts (arrow) and a mucin plug (arrowhead). f-h Histopathological images show mucin-producing epithelial cells without nuclear atypia forming a papillary arrangement, which was diagnosed as IPMN with low-grade dysplasia [HE staining; original magnification $\times 10(\mathbf{f}, \mathbf{g}), \times 20(\mathbf{h})]$. The mucin plug is confirmed (arrowhead)

and $64-88 \%$, respectively [59]. Further EUS examination should be performed without hesitation.

On the other hand, SPN has a cystic nature, with the solid component composed of uniform, polygonal epithelioid cells with vascularized stroma that are not cohesive in arrangement and show slow fill-in enhancement after contrast administration. The presence of solid components does not necessarily indicate malignant transformation [53].

Enhancement of the numerous septations of microcystic serous cystadenomas on CT can give the misleading appearance of partly solid components (Fig. 4), although the finding of a cluster of small fluid-containing cysts on MRI is usually diagnostic.

Lymphoepithelial and epidermoid cysts often contain granular keratinized material that may be depicted as mural nodules. This material is visualized as slight hyperdensity on CT, and as hypointensity on T2-weighted MRI and hyperintensity on T1-weighted MRI (Fig. 15) [55]. In addition, the non-degenerated area of an epidermoid cyst within intrapancreatic accessory spleen may be depicted as a solid component, but can show intensity on MRI and have an enhancement pattern similar to that of splenic parenchyma $[25,26]$. 


\section{Fluid content}

On CT, non-complicated serous or mucinous cystic lesions are depicted as lesions with water density (0-15 Hounsfield units), whereas on MRI, they have homogeneous hypointensity on T1-weighted images and hyperintensity on $\mathrm{T} 2$-weighted images. Therefore, the density or intensity of the contents cannot discriminate mucinous tumors having malignant potential from serous benign lesions (Figs. 1, 5).

In some cases of MCN, SPN, and pseudocyst, however, the content does not show typical water density or intensity. These lesions may contain sullage or hemorrhage that can affect the signal intensity depending on the amount that is present $[7,10,13,60]$. Furthermore, a fluid-fluid level (in cystic areas) or fluid-debris level (in areas of hemorrhagic degeneration) may be present in some cases (Fig. 13) [28, 52].

Lymphoepithelial cysts contain granular keratinized material or lipid component. The density on unenhanced CT or signal intensity on MRI varies depending on the amount of keratinized material or lipid component: these lesions sometimes show hyperdensity on non-contrast CT or hyperintensity on T1-weighted MRI (Fig. 14) [55]. Epidermoid cysts also contain various amounts of keratinized material, and the contents can have a similar appearance to that of lymphoepithelial cysts (Fig. 15) [25, 26].

\section{Calcification}

Morphology and frequency of calcification are key findings of pancreatic cystic lesions, although these are not critical in the differential diagnosis.

A characteristic stellate pattern of calcification with a fibrous central scar is seen in $30 \%$ of serous cystadenomas, and is highly specific and pathognomonic for serous cystadenoma [61].

Although peripheral eggshell calcification is seen infrequently (in 15\% of cases), it is specific for MCN and is highly predictive of malignancy [62].

Calcification is common in SPN, being reported in 30\% of cases, and is characteristically peripheral and punctate [63]. There is no substantial difference in calcification pattern between benign and malignant SPNs.

Calcification is reported in $20 \%$ of IPMNs. Punctate calcification is the most common pattern $(87 \%)$, followed by coarse calcification (33\%). The presence, pattern, or location of calcification in IPMN has no correlation with malignancy

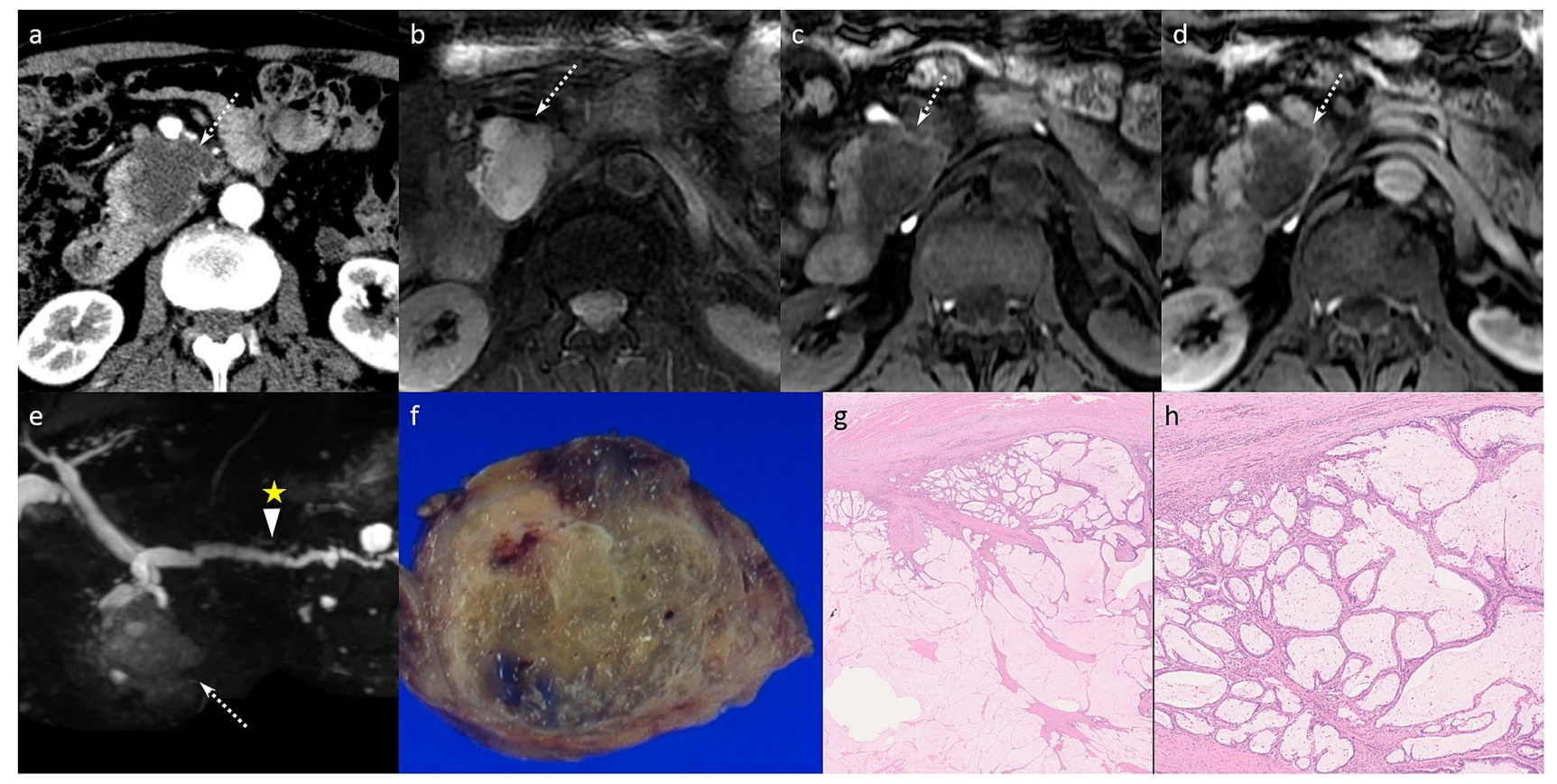

Fig. 8 An 80-year-old man with a cystic lesion in the pancreatic head mimicking pseudocyst that was diagnosed surgically as branch duct intraductal papillary mucinous carcinoma (IPMC). The indistinct wall and irregular shape of the lesion makes diagnosis difficult. a Portal phase CT shows an irregularly shaped cystic lesion (arrow) of the pancreatic head, approximately $3 \mathrm{~cm}$ in diameter. There is no evidence of wall thickening or mural nodule. $\mathbf{b}$ The lesion shows obvious hyperintensity (arrow) on T2-weighted fat-suppressed MRI. Wall thickening or mural nodule are not seen. c The lesion shows homogeneous hypoin- tensity on precontrast MRI. d No wall thickening or mural nodule are seen on the delayed phase image of dynamic contrast MRI. e The cystic lesion (arrow) can be visualized but appears slightly indistinct on MR cholangiopancreatography. The mild MPD dilatation (arrowhead) upstream of the tumor is probably age related. $\mathbf{f}$ The resected specimen shows the cystic nature of the lesion. $\mathbf{g}$, $\mathbf{h}$ Histopathological images reveal mucin-producing epithelial cells with nuclear atypia forming a papillary arrangement, which is diagnostic for invasive IPMC [HE staining; original magnification $\times 10(\mathbf{g}), \times 20(\mathbf{h})]$ 


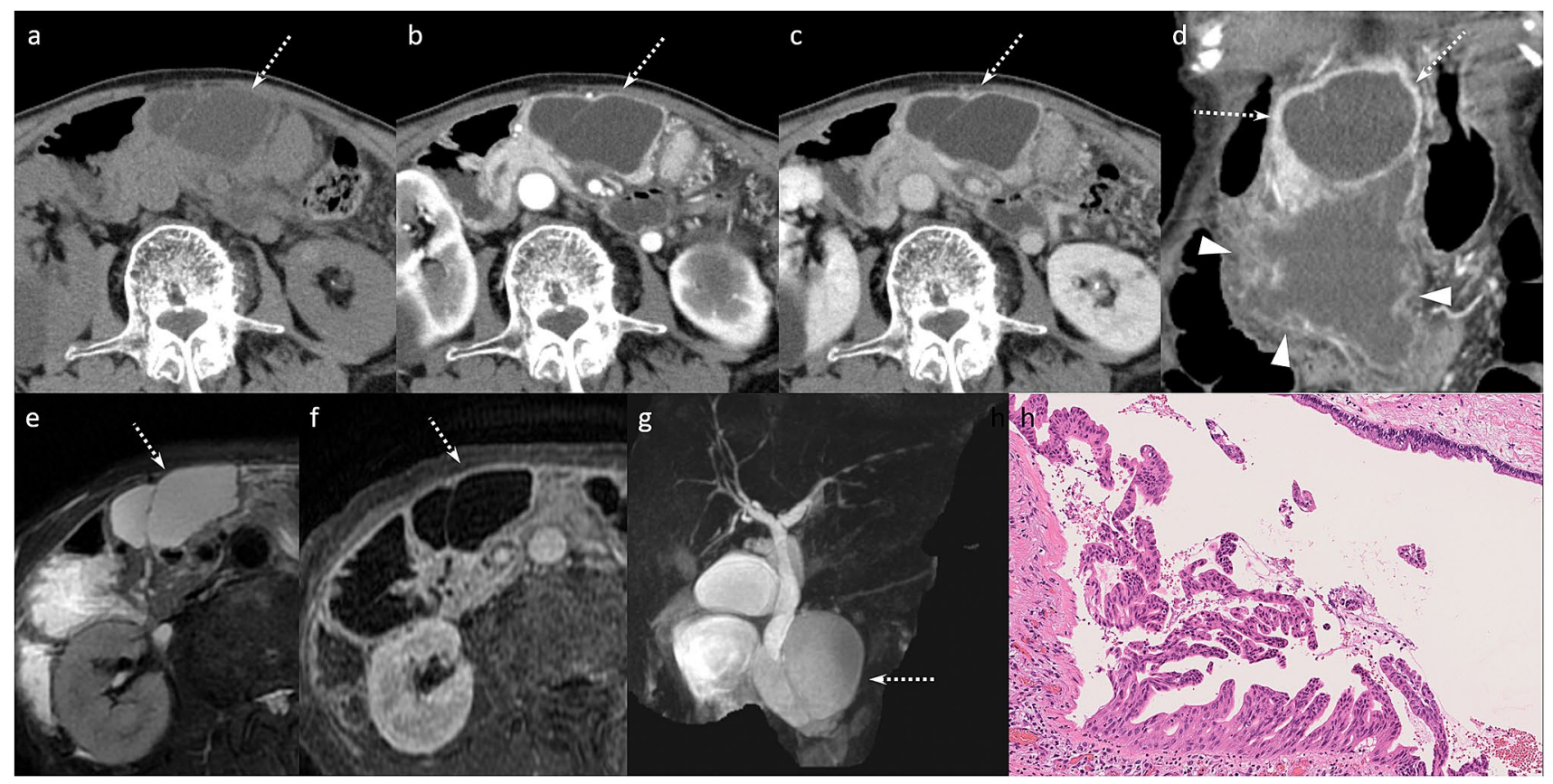

Fig. 9 A 65-year-old man with a cystic lesion in the pancreatic head mimicking pseudocyst and walled-off necrosis after acute pancreatitis, which was diagnosed surgically as ruptured branch duct IPMC. IPMN sometimes ruptures, causing peritonitis, and the patient presents with clinical symptoms similar to those of acute pancreatitis. After rupture, the tension of the lesion is lost. a Unenhanced CT reveals a cystic lesion (arrow) with septa and without calcification. The lesion shows a lack of tension. b, c Arterial (b) and equilibrium (c) phase CT images show a unilocular cystic lesion (arrows) and no irregularity of the wall or mural nodules. d Coronal portal phase of dynamic contrasted CT reveals the unilocular cystic lesion (arrows) and an ill-defined caudal fluid collection (arrowheads). e T2-weighted fat-suppressed MRI shows the cystic lesion (arrow) without tension. f Delayed phase image of dynamic contrasted MRI shows no enhancement of the lesion (arrow). g MR cholangiopancreatography depicts the cystic lesion (arrow). h Histopathological images reveal mucin-producing epithelial cells with nuclear atypia forming a papillary arrangement, which is diagnostic for invasive IPMC (HE staining; original magnification $\times 40$ )

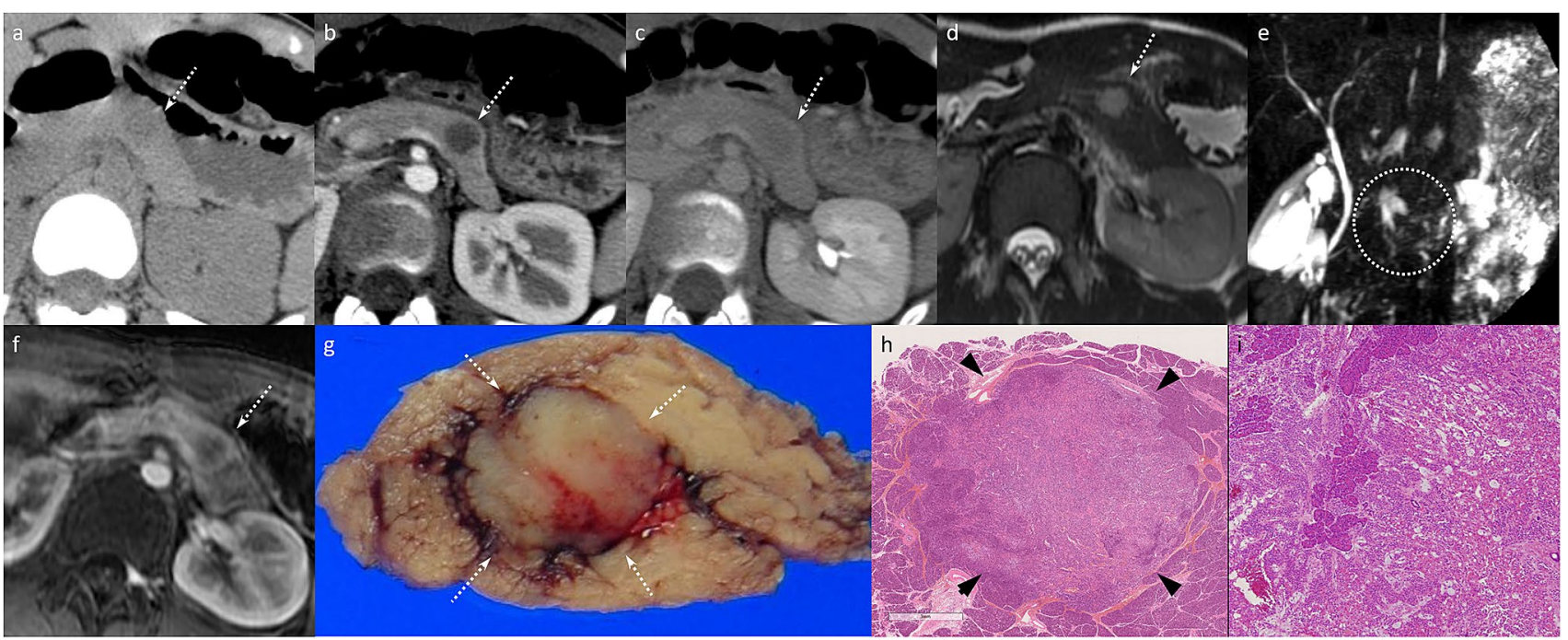

Fig. 10 A 15-year-old girl with a round lesion in the pancreatic tail. The lesion mimics pseudocyst accompanied by hemorrhage, and was diagnosed surgically as a solid pseudopapillary neoplasm. Degeneration of a solid mass shows hyperintensity on T2-weighted imaging that can resemble that of inherently cystic lesions. Note that the imaging findings of SPN can closely resemble those of pseudocyst (see Fig. 11), and it can be difficult to discriminate between them. a Unenhanced CT depicts an approximately $1-\mathrm{cm}$-diameter, slightly hypodense mass (arrow) in the pancreatic tail. b No enhancement of the lesion is seen on portal phase CT (arrow). c No enhancement of the lesion is observed on equilibrium phase CT (arrow) relative to the unenhanced CT image. d The lesion shows obvious hyperintensity (arrow) on T2-weighted MRI. e The lesion is indistinct (dotted circle) on MR cholangiopancreatography. $\mathbf{f}$ The lesion (arrow) shows no enhancement on a portal phase image of dynamic contrast MRI. $\mathbf{g}$ The resected specimen shows a solid mass with hemorrhage (arrows). h, i Histologically, the tumor (arrowheads) is composed of uniform round cells with a moderate amount of eosinophilic cytoplasm and mild nuclear atypia. The cells tend to be poorly cohesive and fall apart, creating pseudopapillary and cystic areas [HE staining; original magnification $\times 5(\mathbf{h}), \times 20(\mathbf{i})]$ 


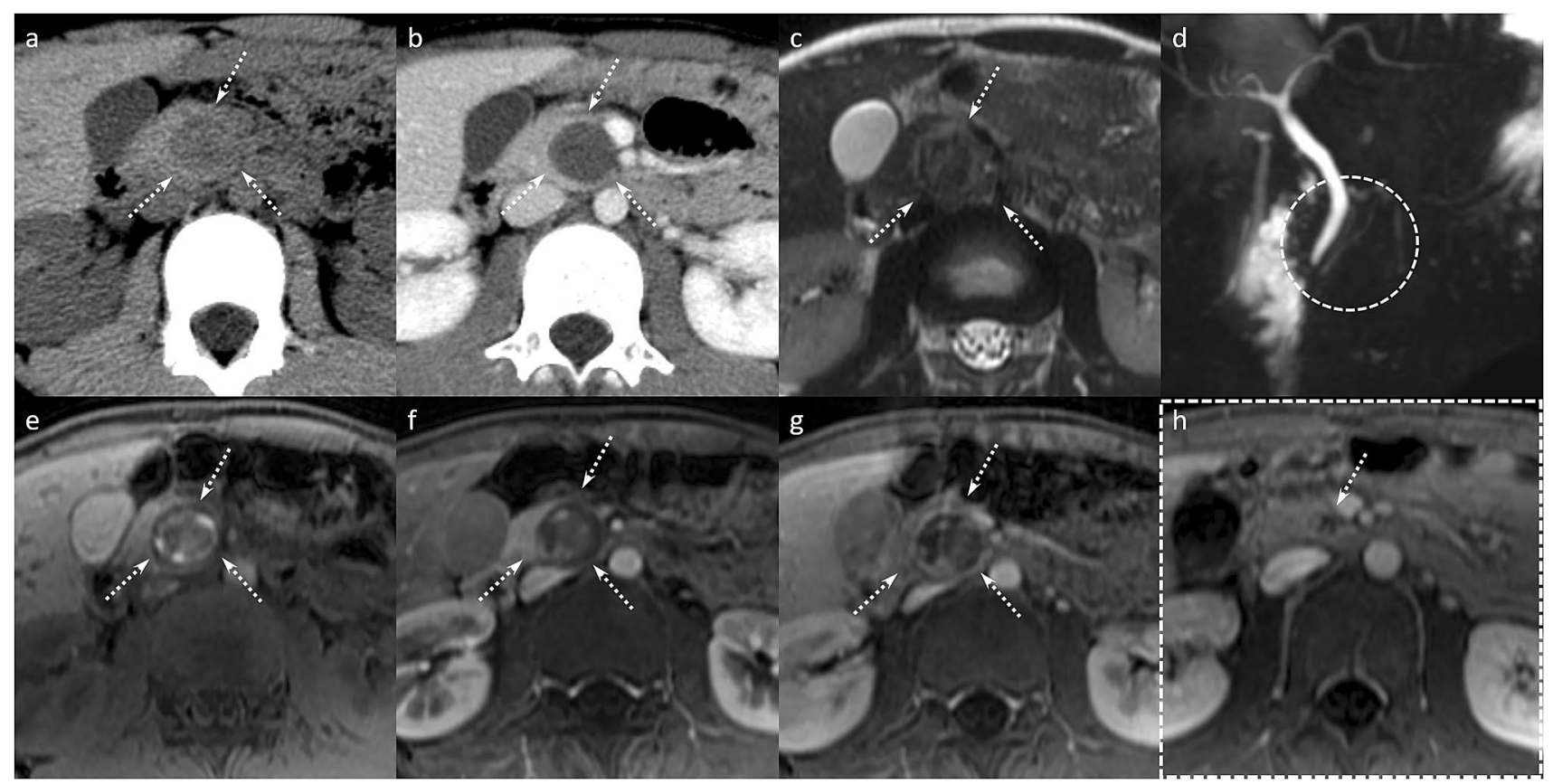

Fig. 11 A 12-year-old girl with a lesion in the pancreatic head mimicking a solid pseudopapillary tumor. The lesion was diagnosed as pseudocyst accompanied by hemorrhage, which can be indistinct on MR cholangiopancreatography. Diagnosing pseudocyst is difficult if there is no history of acute or chronic pancreatitis. Note that the imaging findings of pseudocyst sometimes closely resemble those of SPN (see Fig. 10) and it can be difficult to discriminate between them. Short-term observation is sometimes helpful for the differential diagnosis. a Unenhanced CT depicts a 2-cm-diameter mass (arrows) showing hypo-density compared with pancreatic parenchyma. b Equilibrium phase CT shows no enhancement of the mass (arrows).

$[64,65]$. Coarse calcification was reported to be associated with malignancy in $>80 \%$ of cases, but these lesions had coexisting malignant features, such as main-duct dilatation, solid nodules, or size $>3 \mathrm{~cm}$ [65].

Calcification is found in up to $16 \%$ of neuroendocrine tumors, arising from cystic necrosis with subsequent development of dystrophic calcification. They tend to be focal, coarse, irregular, and centrally located [66], and occur more commonly within non-functioning and larger neoplasms [61].

In other tumors, calcifications within the lesion are rare. c The mass shows inhomogeneous hypointensity on T2-weighted MRI (arrows), and hemorrhagic content is suspected. d The mass is not depicted on MR cholangiopancreatography (dotted circle). e The mass shows inhomogeneous hyperintensity on precontrast T1-weighted fat-suppressed MRI (arrows), and hemorrhagic content is also suspected. f, $\mathbf{g}$ Arterial (f) and portal (g) phase images of dynamic contrast study show no enhancement of the lesion (arrows). The areas of inhomogeneous hyperintensity have the same appearance as on the precontrast image. $h$. Delayed phase image obtained after 3 months shows shrinkage of the lesion (arrow)

\section{Localization (most frequent)}

Localization within the pancreas may give some clues for the differential diagnosis. Approximately $40 \%$ of pancreatic serous cystadenomas arise from the pancreatic head and uncinate process, and $60 \%$ arise from the pancreatic body and tail [67]. Conversely, MCN occurs most commonly in the pancreatic tail [27], and rarely in the pancreatic head. SPN arises predominantly in the pancreatic tail [63].

Branch duct IPMN is typically located within the pancreatic head, preferentially within the uncinate process [15]. In up to two-thirds of cases, however, smaller cysts can also be found in other parts of the pancreas [68].

Neuroendocrine tumors can have predominantly exophytic growth [69]. Those with cystic changes tend to be 


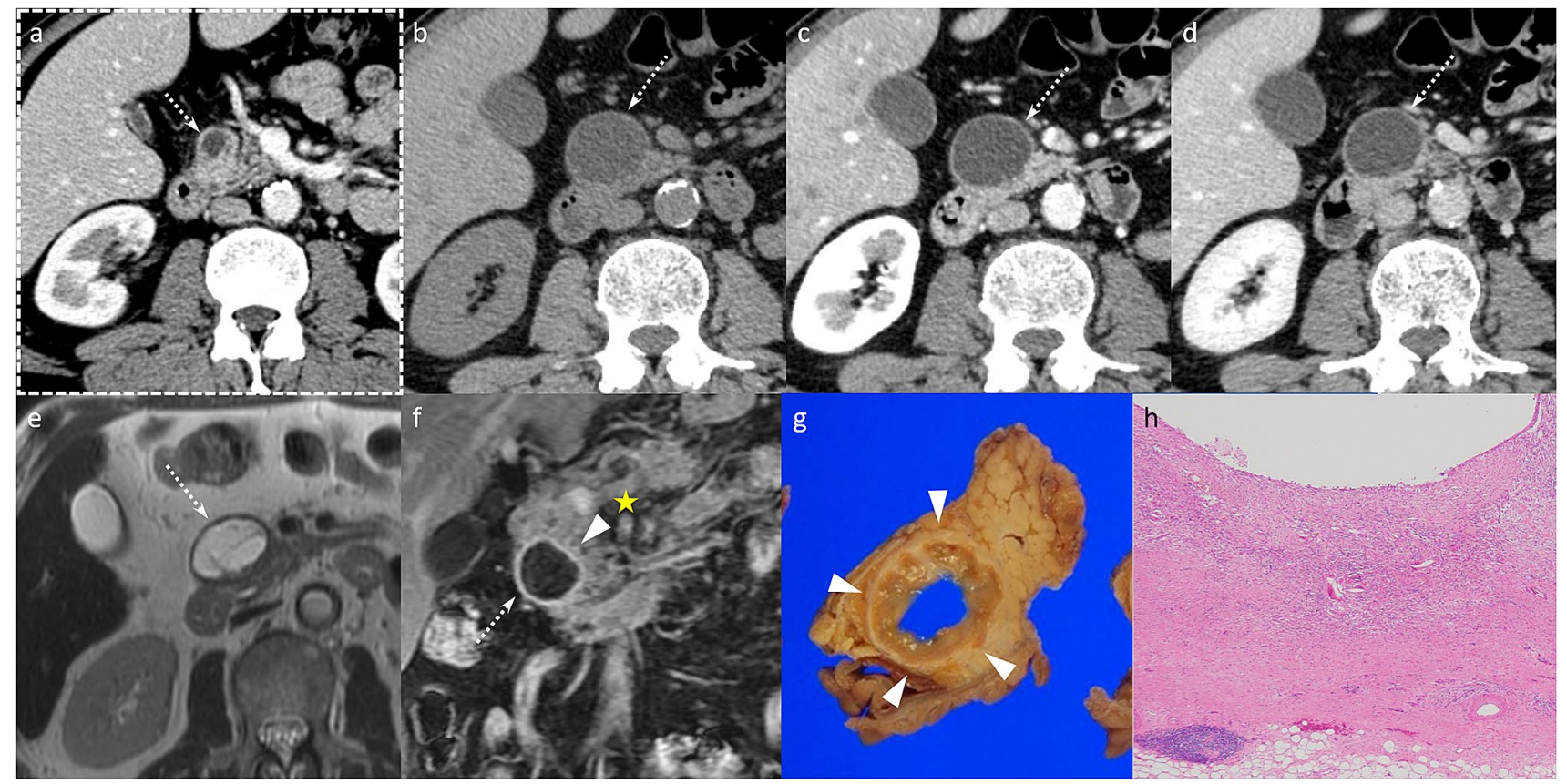

Fig. 12 A 63-year-old man with a unilocular cystic lesion in the pancreatic head mimicking macrocystic serous cystadenoma or lymphoepithelial cyst, which was diagnosed surgically as pseudocyst. Pseudocyst can show inflammatory wall thickening with homogeneous enhancement. An obscure history of pancreatitis and enlargement during long-term observation increase the difficulty of diagnosing pseudocyst. a Portal phase CT obtained 3.5 years previously shows a small cystic lesion (arrow) in the pancreatic head, approximately $0.8 \mathrm{~cm}$ in diameter. b Unenhanced CT reveals the same unilocular cystic lesion in the head (arrow), without calcification, now enlarged to approximately $5 \mathrm{~cm}$ in diameter. c Portal phase CT reveals indis-

located in the body or tail of the pancreas, whereas those without cystic changes are significantly more likely to be located in the head $[24,70]$.

Lymphoepithelial cysts are mainly extrapancreatic or exophytic in nature $[54,55]$. Epidermoid cyst in intrapancreatic accessory spleen is located exclusively in the tail of the pancreas $[25,26]$. Pseudocyst can occur in any part of the pancreas [36].

\section{Number of lesions}

Most pancreatic cystic lesions are basically unifocal. Multifocal lesions are rare, particularly in MCN and SPN. Lymphoepithelial and epidermoid cysts are also commonly unifocal. tinct wall thickening (arrow). d Equilibrium phase CT shows a unilocular cystic lesion in the head with slight wall thickening (arrow). e T2-weighted MRI depicts the wall as homogeneous and slightly thickened (arrow). f Coronal phase image of dynamic contrasted MRI depicts the unilocular cystic lesion (arrow) with homogeneous enhancement of the thickened wall (arrowhead). $\mathbf{g}$ The resected specimen shows a unilocular cystic lesion with uniform thickening of the wall (arrowheads; original magnification $\times 10$ ). $\mathbf{h}$ Histopathological image reveals fibrocollagenous wall with inflammation and no lining epithelium (HE staining)

Branch duct IPMN is often multifocal, with rates perhaps as high as $25 \%$ [16]. It is reported that multifocal IPMN can indicate a higher risk of malignancy [71]. The risk of malignant transformation is reported as $58.8 \%$ for multifocal IPMN and 49.2\% for unifocal IPMN [71]. Although not initially visualized on cross-sectional imaging, additional invasive or noninvasive IPMN can develop in the pancreatic remnant after resection of a noninvasive IPMN [72].

Pancreatic cystic lesions can occur in genetic diseases, such as Von Hippel-Lindau disease, multiple endocrine neoplasia (MEN) type 1, cystic fibrosis, and autosomaldominant polycystic kidney disease (ADPKD). Pancreatic cystic lesions associated with these genetic disorders can be highly multifocal. Among these, cellular dysfunction may affect part or most of the exocrine or endocrine pancreatic 


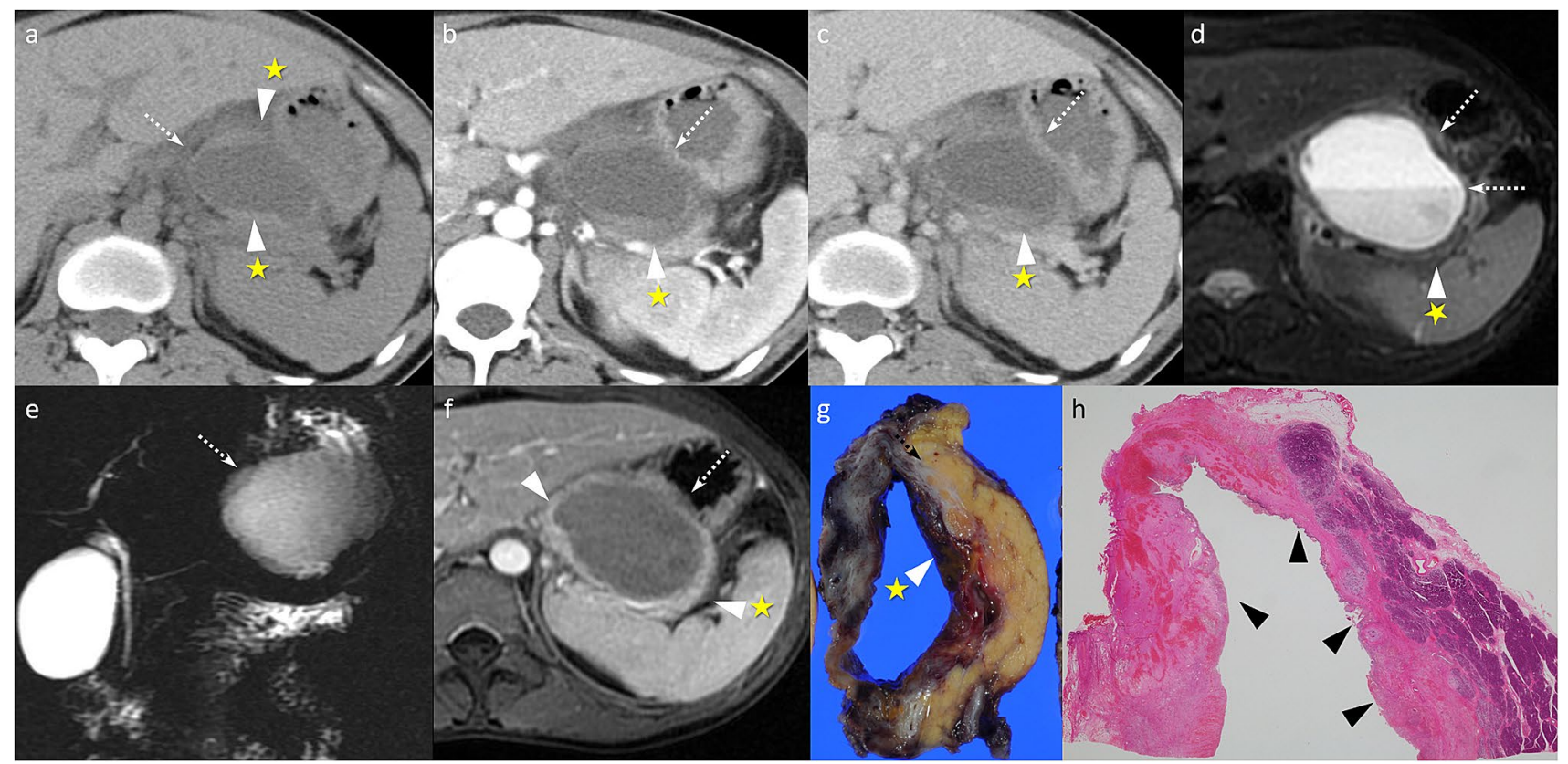

Fig. 13 A 25-year-old woman with a unilocular cystic lesion mimicking solid-pseudopapillary neoplasm that was diagnosed surgically as pseudocyst accompanied by hemorrhage. Pseudocyst with inflammation can show irregular wall thickening, and hemorrhage within the lesion that is depicted as a fluid-fluid level. Diagnosing pseudocyst is difficult if there is no history of pancreatitis. a Unenhanced CT shows a unilocular cystic lesion that has a lack of tension (arrow) and irregular wall thickening (arrowheads). b, c Arterial (b) and equilibrium (c) phase CT images show the unilocular cystic lesion (arrows) and irregular wall thickening (arrowheads). d T2-weighted fat-suppressed MRI reveals a fluid-fluid level within the unilocular cystic lesion,

cells and lead to the development of multiple and various pancreatic cystic lesions. As these cysts generally share the imaging and histological features of their non-inherited counterparts, they share the same diagnostic procedure, management, and follow-up.

Multiple serous cystadenomas can occur in von Hippel-Lindau disease [73]. Simple cysts or neuroendocrine tumor with or without cystic degeneration can also occur, and in some patients, cystic growth replaces almost the entire pancreas [74]. Although other causes of multifocal pancreatic cyst are less common, $36.4 \%$ of cases of ADPKD are reported to have at least one pancreatic cyst [75].

Most neuroendocrine tumors with or without cystic changes $(>90 \%)$ are sporadic and $10 \%$ are associated with genetic syndromes, such as MEN type 1, von Hippel-Lindau disease, neurofibromatosis type 1 , and tuberous sclerosis $[45,75]$. The most common pancreatic neoplasms in MEN1 are gastrinoma and insulinoma $[45,75]$. indicative of hemorrhage (arrows). Inhomogeneous wall thickening is observed (arrowhead). e The lesion is ill-defined on MR cholangiopancreatography (arrow). No dilatation of the MPD or communication with the MPD is seen. $\mathbf{f}$ Delayed phase image of dynamic contrasted MRI depicts the unilocular cystic lesion (arrow) and irregular thickening of the wall (arrowheads). $g$ The resected specimen shows a unilocular cystic lesion with homogeneous wall thickening and hemorrhage (arrowhead). h Loupe image reveals a fibrocollagenous wall with inflammation, hemorrhage and no lining epithelium (arrowheads) (HE staining)

\section{Original size and change in size over time}

Original size and change in size over time are also key elements in differential diagnosis.

The median size of MCNs is $5 \mathrm{~cm}$ and $61 \%$ are larger than $5 \mathrm{~cm}$ at detection [76]. SPNs are also often relatively large, with a mean size of 7.5-11 cm at diagnosis [63].

Serous cystadenomas vary in size at diagnosis and size is generally stable over time. Some cases are slow-growing (Figs. 1, 2). Progressive enlargement of serous cystadenomas, especially those $\geq 4 \mathrm{~cm}$ at the initial manifestation, can be observed over a period of months or years [77].

In branch duct IPMN, size is a clear indicator of malignancy. Branch duct IPMN $<3 \mathrm{~cm}$ in size and without other risk factors can be managed conservatively [16]. Side-branch IPMN should be followed up carefully for "worrisome features", such as cyst enlargement $>3 \mathrm{~cm}$, thickened and 


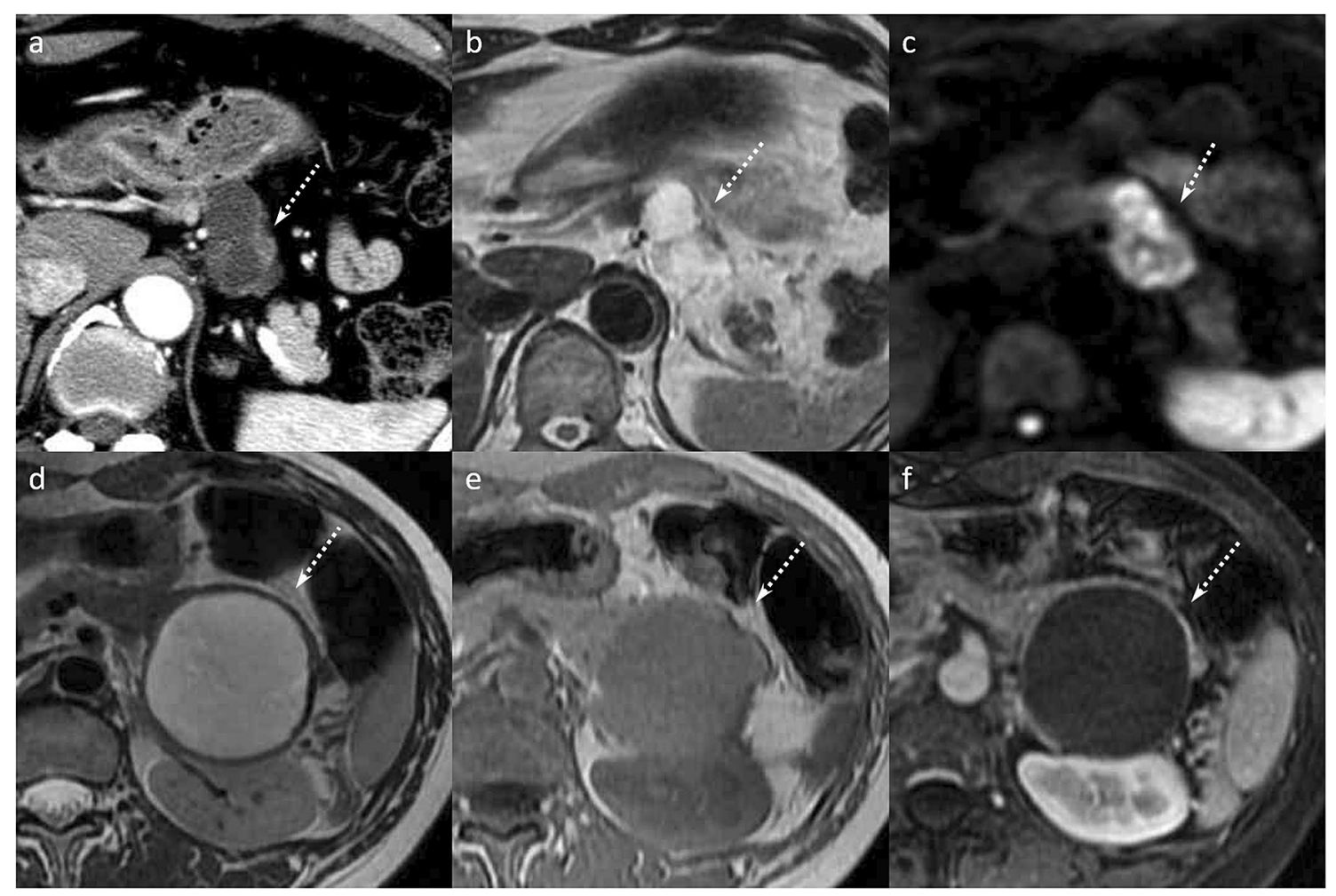

Fig. 14 a-c A 56-year-old man with an exophytic cystic lesion in the pancreatic body mimicking serous cystadenoma or MCN that was diagnosed surgically as lymphoepithelial cyst. a Arterial phase CT reveals an exophytic and multilocular cystic lesion in the body (arrow) with slight wall thickening. b The lesion shows clear hyperintensity on T2-weighted MRI (arrow). c The lesion shows inhomogeneous hyperintensity on diffusion-weighted MRI (arrow). d-f A

enhancing cyst wall, enhancing mural nodule $<5 \mathrm{~mm}$, or MPD 5-9 $\mathrm{mm}$ in diameter. A lesion $>3 \mathrm{~cm}$ with or without MPD dilatation of $>10 \mathrm{~mm}$ is strongly suggestive of malignancy [16].

When there is significant overlap of imaging features between IPMN and serous cystadenoma, serial follow-up imaging evaluations are helpful because pancreatic pseudocysts often evolve over short intervals, whereas other pathologies can persist without significant short-term change.

In contrast, some studies have not reported a precise diagnosis due to limitations of the cross-sectional imaging findings. Small cystic pancreatic lesions $(<3 \mathrm{~cm}) \mathrm{com}-$ monly have no diagnostic imaging features at all, whereas there can be a substantial overlap of findings between larger mucinous and nonmucinous cystic pancreatic 49-year-old woman with a cystic lesion in the pancreatic tail mimicking mucinous cystadenoma that was diagnosed surgically as lymphoepithelial cyst. d T2-weighted MRI reveals a unilocular cystic lesion (arrow) that has slightly thickened and homogenous wall. e The cyst contents show slight hyperintensity on T1-weighted MRI (arrow). f Delayed phase image of dynamic contrasted MRI shows enhancement of the thickened wall (arrow)

lesions [78]. Many authors have suggested tumor sizes that may be associated with a higher risk of malignancy $[29,37,79]$, and size is a well-known criterion in the selection of patients suitable for surgery. One of these studies suggested that patients with small $(<3 \mathrm{~cm})$ and asymptomatic cysts without solid components should be followed up radiographically as part of assiduous followup, as resection should be performed if changes occur within the cyst or if the patient develops symptoms [79]. Unsurprisingly, others have recommended taking additional factors into account. For example, Sakorafas et al. [29] recommend a more aggressive treatment policy for cystic tumors that are located in the body or tail of the pancreas for the reason that the surgical procedure is less destructive than for lesions in the head. Buscaglia et al. 


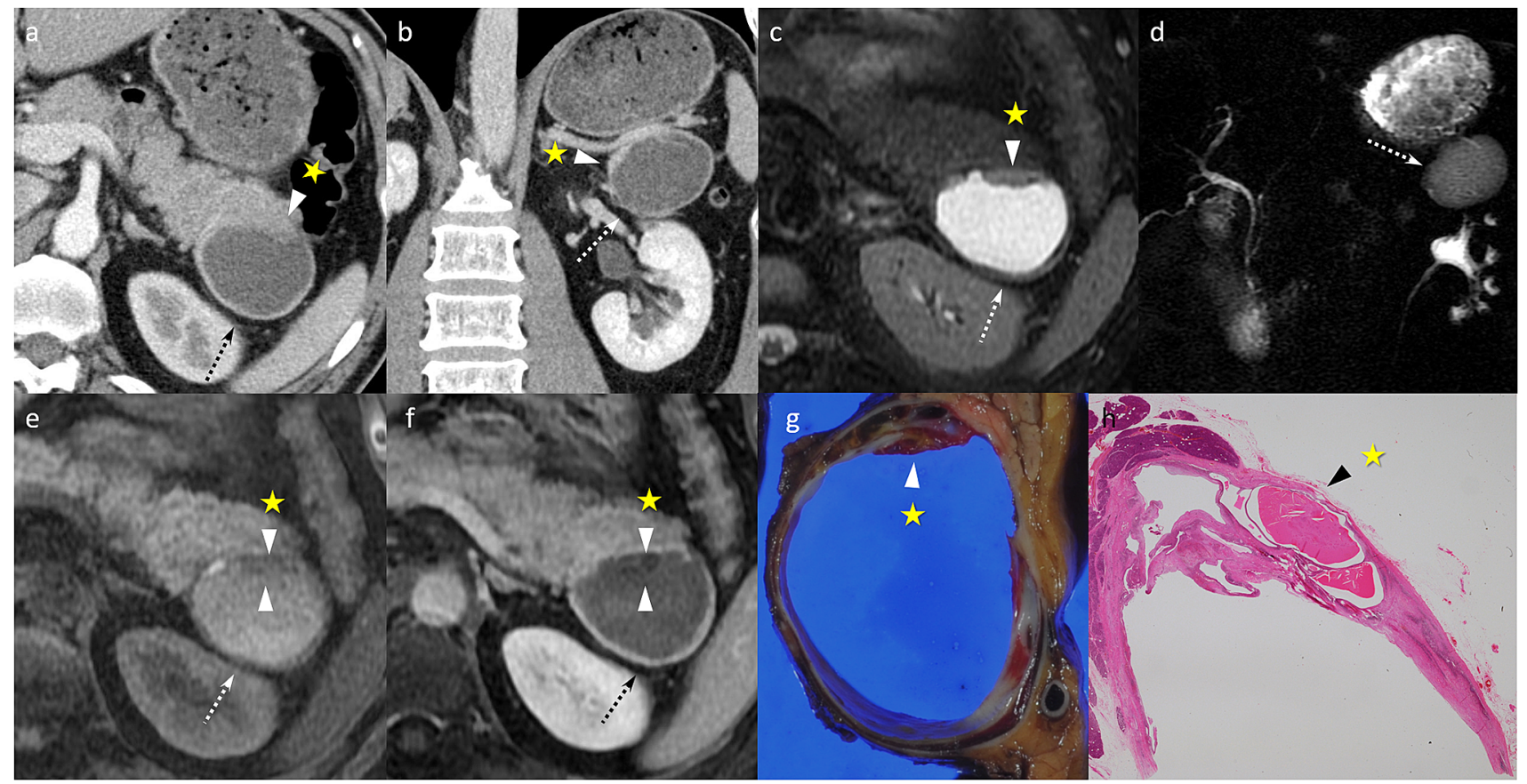

Fig. 15 A 57-year-old man with a unilocular cystic lesion mimicking mucinous cystic neoplasm that was diagnosed surgically as an epidermoid cyst of intrapancreatic accessory spleen. Epidermoid cysts can show wall thickening and mural nodule-like lesions. a, b Axial (a) and coronal (b) equilibrium phase CT show an approximately 5-cm-diameter, unilocular cystic lesion in the pancreatic tail (arrows). Irregular thickening of the cystic wall is observed (arrowheads). c T2-weighted fat-suppressed MRI shows inhomogeneous thickening of the wall (arrowhead) within the cystic lesion (arrow). d MR cholangiopancreatography depicts a relatively ambiguous cystic lesion

[80] attempted to develop a predictive model for pancreatic cyst malignancy using the CEA level in fluid content, in addition to age $>50$ years and cyst size $>1.5 \mathrm{~cm}$. Other studies have suggested different patient management based on combinations of clinical characteristics, such as age, gender, presence of symptoms, cyst size and location, and comorbidities [1,81,82].

\section{Pancreatic parenchymal change and peripancreatic findings}

\section{Dilatation of the main pancreatic duct}

When a pancreatic cystic lesion is accompanied by a dilated MPD, the cause is either tumorous dilatation suggesting a main duct or mixed IPMN, or lesion-related dilatation. (arrow). e Precontrast T1-weighted fat-suppressed MRI reveals inhomogeneous hyperintensity of the cystic contents (arrow), suggestive of sullage or hemorrhage. f Delayed phase image of dynamic contrasted MRI reveals the unilocular cystic lesion with wall thickening (arrow) and a mural nodule-like lesion that shows no enhancement (arrowhead). $\mathbf{g}$ The resected specimen shows a unilocular cystic mass with a mural nodule-like lesion (arrowhead). h Loupe image reveals a unilocular cyst with stratified squamous epithelium and keratinous material (arrowhead) (HE staining)

Main duct IPMN is characterized by segmental or diffuse MPD dilation ( $>5 \mathrm{~mm}$ ) with no other cause of obstruction. MPD dilation of 5-9 $\mathrm{mm}$ is considered a "worrisome feature" and MPD diameter of $10 \mathrm{~mm}$ is a "high-risk stigmata". Mixed-type patients meet the criteria for both main and branch duct IPMNs [16].

Lesion-related MPD dilation is further divided into two conditions, MPD compression and chronic pancreatitis of the background parenchyma. Most serous cystadenomas do not show MPD dilation, as they are rarely accompanied by upstream MPD dilatation caused by a mass effect due to extrinsic compression (Figs. 2, 3, 4) [83]. In detail, MPD dilatation, which is mostly associated with mucin secreted from the tumor, is exclusively diffuse in IPMN, whereas MPD dilatation tends to be localized to upstream serous cystadenoma [83]. Some cases of branch duct IPMN and serous cystadenoma have age-related dilatation of the MPD (Figs. 2, 8), 


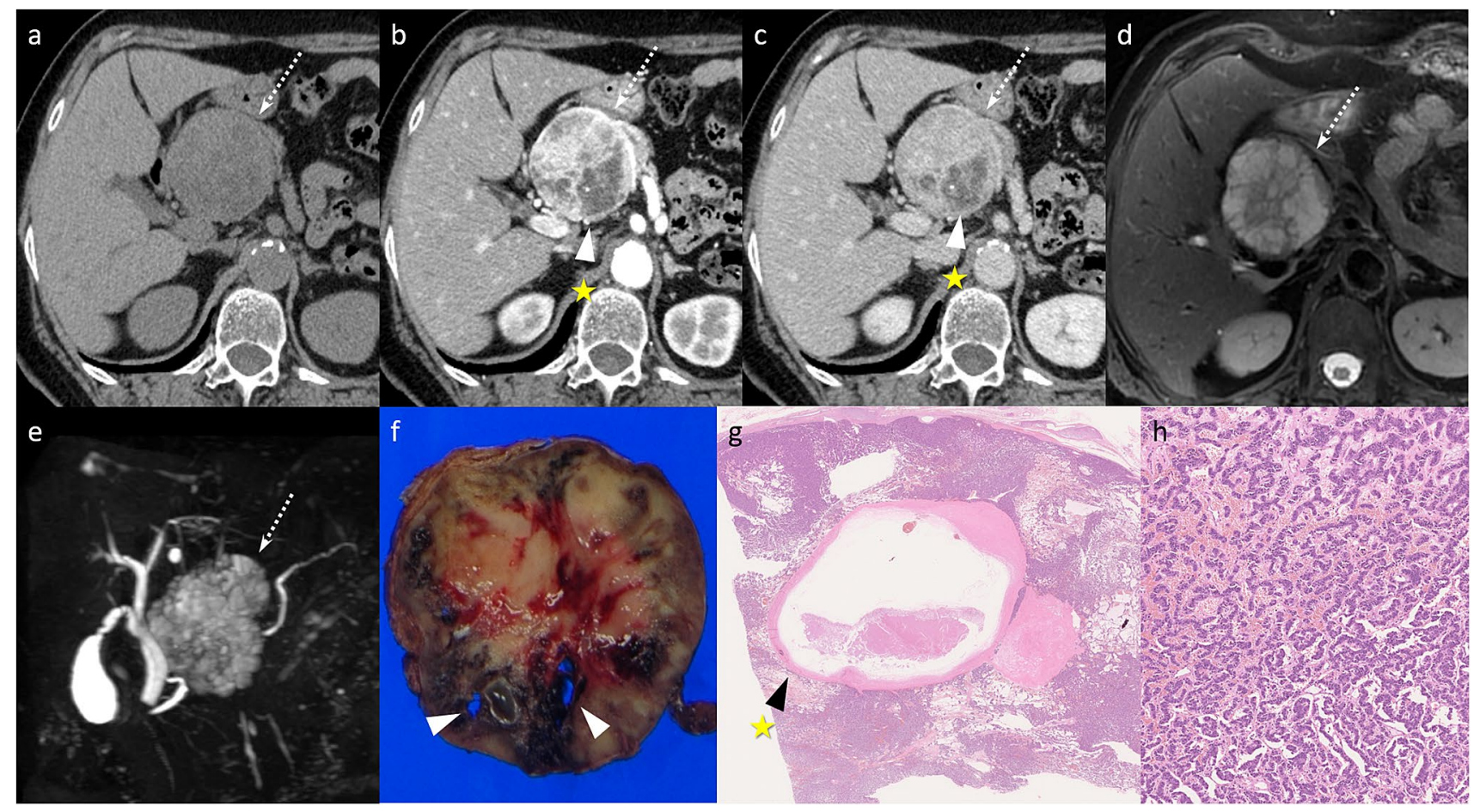

Fig. 16 A 75-year-old woman with a mass in the pancreatic head mimicking mixed micro-and macro-cystic serous cystadenoma that was diagnosed surgically as neuroendocrine tumor (G1). Enhanced CT images indicate the solid portion, however the obvious hyperintensity on T2-weighted and MR cholangiopancreatography images complicates the diagnosis. Note that the imaging findings closely resemble those of Fig. 4 (serous cystadenoma). a Unenhanced CT shows a 5.5-cm-diameter, well-defined, uncalcified mass located in the pancreatic head (arrow). b The mass shows inhomogeneous early enhancement (arrow), and is accompanied by an unenhanced area (arrowhead) on arterial phase CT. c Inhomogeneous enhancement

which is rarely $>5 \mathrm{~mm}$ in diameter [84], and the differential diagnosis from mixed IPMN can be challenging.

Ductal adenocarcinoma inevitably shows MPD dilation upstream of the tumor, which also occurs due to extrinsic compression of the MPD. However, as mentioned in the classification of pancreatic cystic lesions section, ductal adenocarcinoma rarely shows complete cystic configurations that mimic real pancreatic cystic lesions.

Pseudocysts are common in chronic pancreatitis that presents with MPD dilation [8, 48]. In contrast, other cystic lesions, such as SPN and MCN, rarely cause MPD dilatation, even if located in the pancreatic head $[9,13,28]$. (arrow) is also seen on equilibrium phase CT. The mass is accompanied with an unenhanced area (arrowhead). d On T2-weighted fatsuppressed MRI, the mass shows obvious hyperintensity and appears as a cystic lesion with septa (arrow). e The lesion is visualized on MR cholangiopancreatography as a cystic lesion (arrow). f The resected specimen shows a solid mass with cystic degeneration (arrowheads). g Loupe image shows a solid mass with cystic degeneration (arrowhead) (HE staining). h Microscopic image shows a mass consisting of small and uniform cells with round nuclei and cystic degeneration (HE staining; original magnification $\times 20$ )

\section{Communication with the main pancreatic duct}

IPMNs usually communicate with the MPD. This important feature is most clearly depicted on MR cholangiopancreatography [50].

An important differentiating feature between $\mathrm{MCN}$ or serous cystadenomas and IPMN is visualization of pancreatic ductal communication. If a clear channel of communication with the pancreatic duct is visualized, the diagnosis of branch duct IPMN is almost certain because serous cystadenomas and MCN do not communicate with the pancreatic ductal system [50, 85]. In contrast, up to $65 \%$ of pseudocysts may show communication with the ductal system $[36,86]$. 


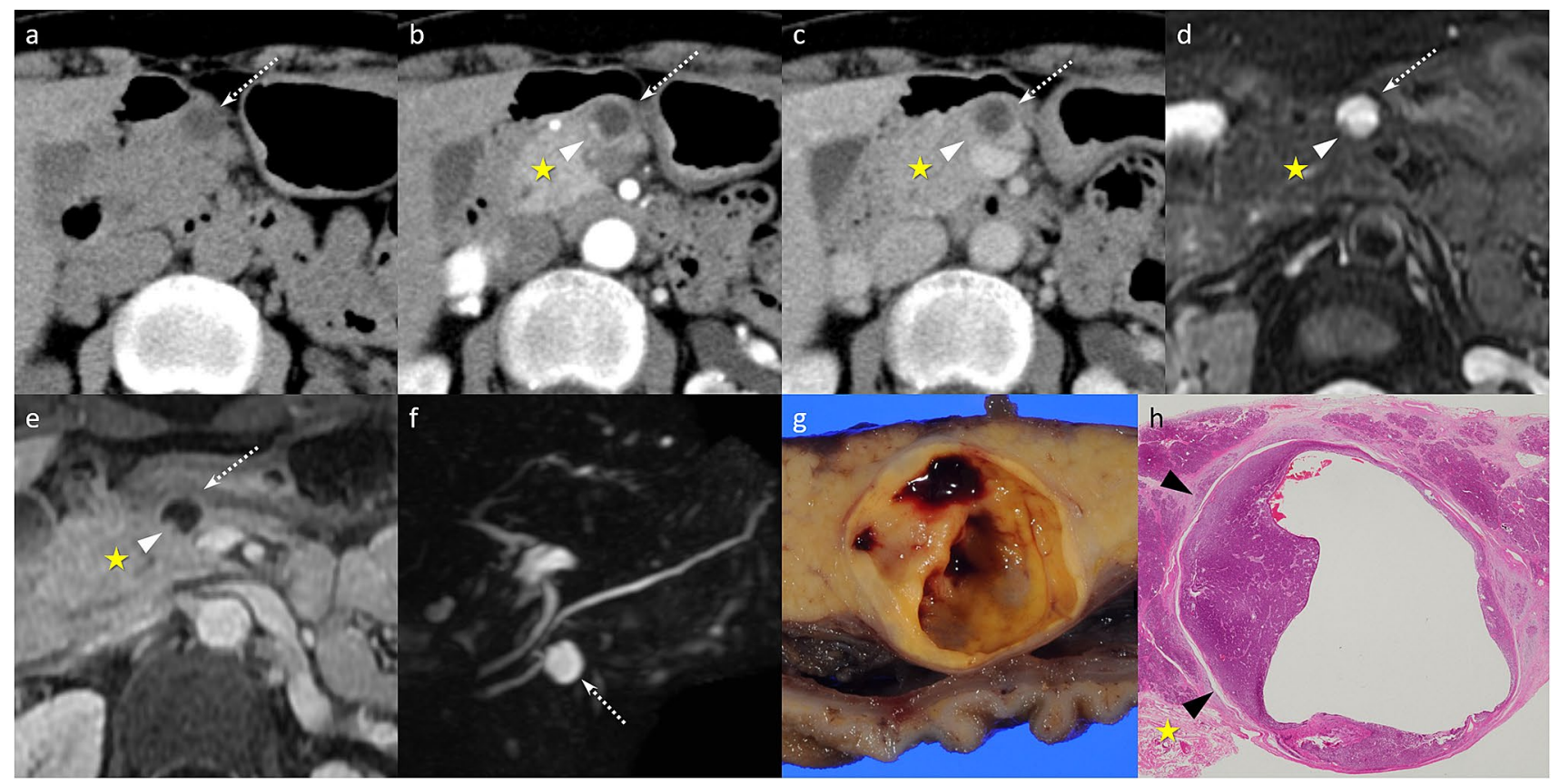

Fig. 17 A 64-year-old woman with a unilocular cystic lesion in the pancreatic head mimicking pseudocyst or small serous cystadenoma that was diagnosed surgically as neuroendocrine tumor (G1). Slight wall thickening and enhancement should not be overlooked. a Unenhanced CT reveals an approximately 1-cm-diameter, uncalcified, unilocular cystic lesion (arrow). b Arterial phase CT shows a unilocular cystic lesion (arrow) with slight wall thickening (arrowhead). c On equilibrium phase CT, the wall thickening (arrowhead) of the lesion (arrow) is less distinct than on the arterial phase image. $\mathbf{d}$ The cystic lesion is seen in the pancreatic head on fat-suppressed T2-weighted

Other cystic lesions, such as MCN and serous cystadenoma, do not usually communicate with the pancreatic ductal system.

\section{Pancreatic parenchymal change and peripancreatic findings}

Typical findings of acute or subacute pancreatitis that are found in pseudocysts include focal or diffuse parenchymal enlargement, changes in density or intensity due to edema, indistinct pancreatic margins due to inflammation, and stranding in the surrounding retroperitoneal fat. MCN and IPMN can uncommonly induce acute pancreatitis (Fig. 5).

Parenchymal atrophy, dilatation of the MPD, and/or calcification suggestive of chronic pancreatitis can be observed in pseudocysts and in ductal adenocarcinoma.
MRI (arrow). A tiny mural nodule or wall thickening is also observed (arrowhead). e Delayed phase image of dynamic contrasted MRI shows slight enhancement of the wall (arrowhead) within the cystic lesion (arrow). f MR cholangiopancreatography clearly depicts a unilocular cystic lesion (arrow). g The resected specimen shows a solid mass with cystic degeneration. h Loupe image shows a solid mass with cystic degeneration. The thickened wall on the radiological images is found to consist of small and uniform cells (arrowheads) (HE staining)

In pseudocysts, findings related to chronic pancreatitis are spread throughout the entire parenchyma, whereas in ductal adenocarcinoma, the findings are located upstream of tumors.

Ruptured IPMN, although rare, has the characteristic finding of peripancreatic fluid collection mimicking pancreatitis (Fig. 9) [87]. It can be challenging to discriminate between ruptured IPMN with fluid collection and pseudocyst with acute pancreatitis. A risk factor for spontaneous rupture of an IPMN could be high pressure if the MPD is filled with mucinous materials, as in our case (Fig. 9), or inflammatory stimulation by factors, such as acute pancreatitis. 


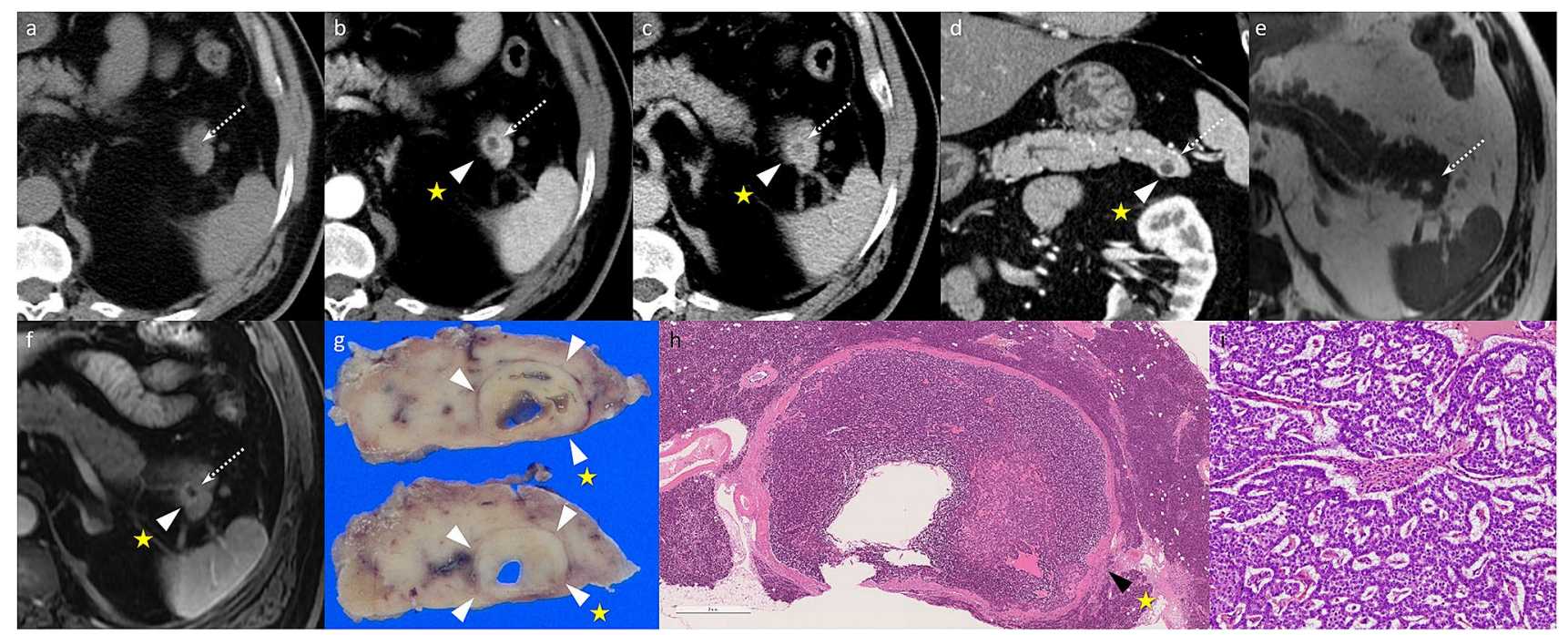

Fig. 18 A 60-year-old man with a cystic lesion mimicking small pseudocyst that was confirmed pathologically as neuroendocrine tumor (Glucagonoma, G1). Neuroendocrine tumor with cystic degeneration can have a similar appearance to lesions that are totally cystic in nature. a Unenhanced CT shows a small unilocular cystic lesion (approximately $5 \mathrm{~mm}$ in diameter), without calcification (arrow). b On arterial phase CT, the lesion shows a similar appearance to a cystic lesion (arrow) and has wall thickening (arrowhead). c, d Axial (c) and coronal (d) equilibrium phase CT also show the unilocular cystic lesion (arrow) and thickening of the wall (arrowhead). e. T2-weighted MRI reveals a tiny and non-specific cystic lesion in the pancreatic tail (arrow). f Delayed phase image of dynamic contrasted MRI shows enhancement of the thickened wall (arrowhead) of the tiny cystic lesion (arrow). $\mathbf{g}$ The resected specimen shows a solid mass with cystic degeneration (arrowheads). h Loupe image shows a solid mass with cystic degeneration (arrowhead) (HE staining). $\mathbf{i}$ The mass consists of small and uniform cells (HE staining; original magnification $\times 20$ )
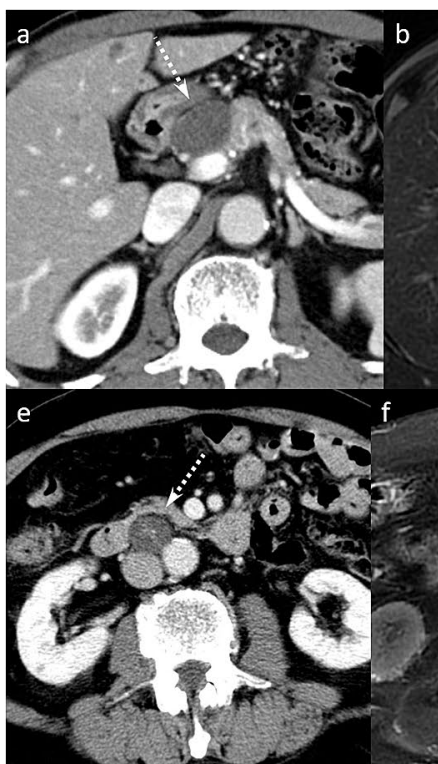
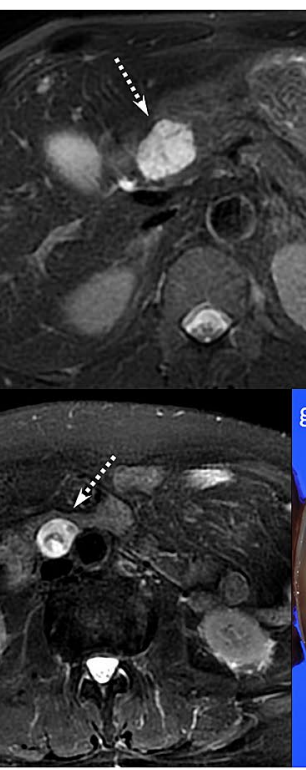
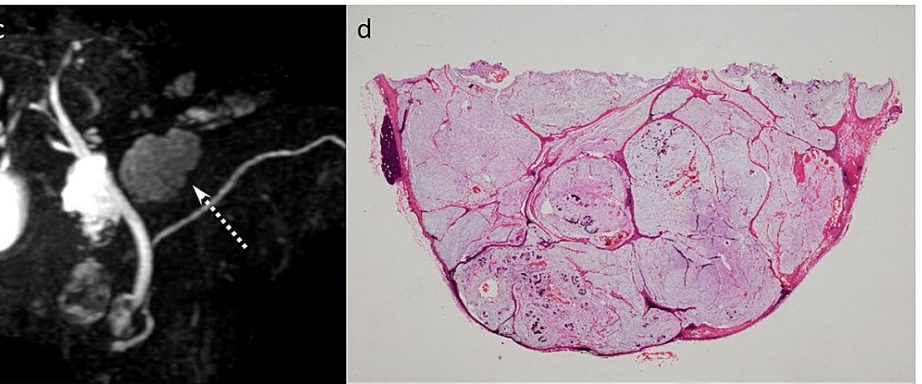

$\mathrm{g}$
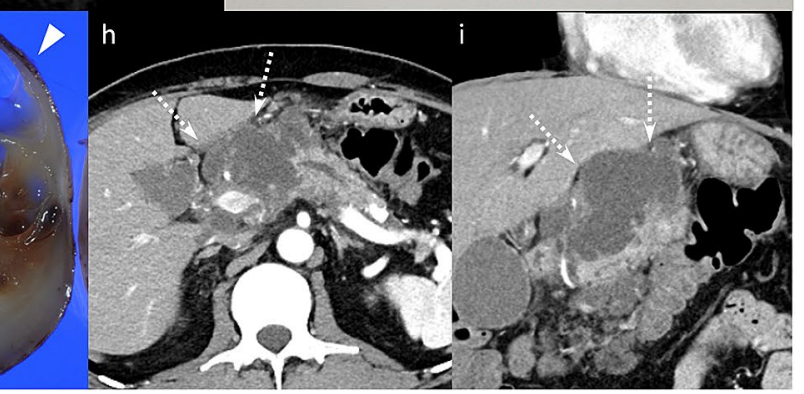

Fig. 19 Lesions that mimic pancreatic cystic lesions. Several peripancreatic lesions are radiologically similar to pancreatic cystic lesions. a-d A 65-yearold man with a cystic lesion in the pancreatic head mimicking branch duct IPMN. The lesion was diagnosed surgically as a metastatic lymph node of mucinous adenocarcinoma. a Portal phase CT reveals a non-enhancing cystic lesion in the pancreatic head. b The mass shows obvious hyperintensity on T2-weighted fat-suppressed MRI. $\mathbf{c}$ The mass is depicted on MR cholangiopancreatography, but is less distinct than the usual appearance of a cystic lesion. d Loupe image reveals that the mass is metastatic lymph node filed with mucin (HE staining). The patient had a history of mucinous adenocarcinoma of the colon. e-g An 83-year-old man with what appears to be a pancreatic cystic lesion as seen in $\mathbf{a}$, which was diagnosed surgically as retroperitoneal schwannoma. Schwannoma can also occur within the pancreatic parenchyma. e Equilibrium phase CT shows an approximately 2-cm-diameter, well-defined, non-enhancing mass adjacent to the pancreatic head (arrow). $\mathbf{f}$ The mass shows obvious hyperintensity on T2-weighted fatsuppressed MRI (arrow). Internal line-like hypointensity is also observed. g The resected specimen shows a solid mass with cystic generation. $\mathbf{h}, \mathbf{i}$ A 62-year-old man with a similar cystic lesion in the pancreatic head as seen in a, mimicking ruptured IPMN. The lesion was diagnosed clinically as walled-off necrosis following acute pancreatitis. The diagnosis of walled-off necrosis after acute pancreatitis is not difficult; however, it is important not to miss a carcinoma or IPMN. h, i Portal phase CT images show ill-defined peripancreatic fluid collection (arrows) 


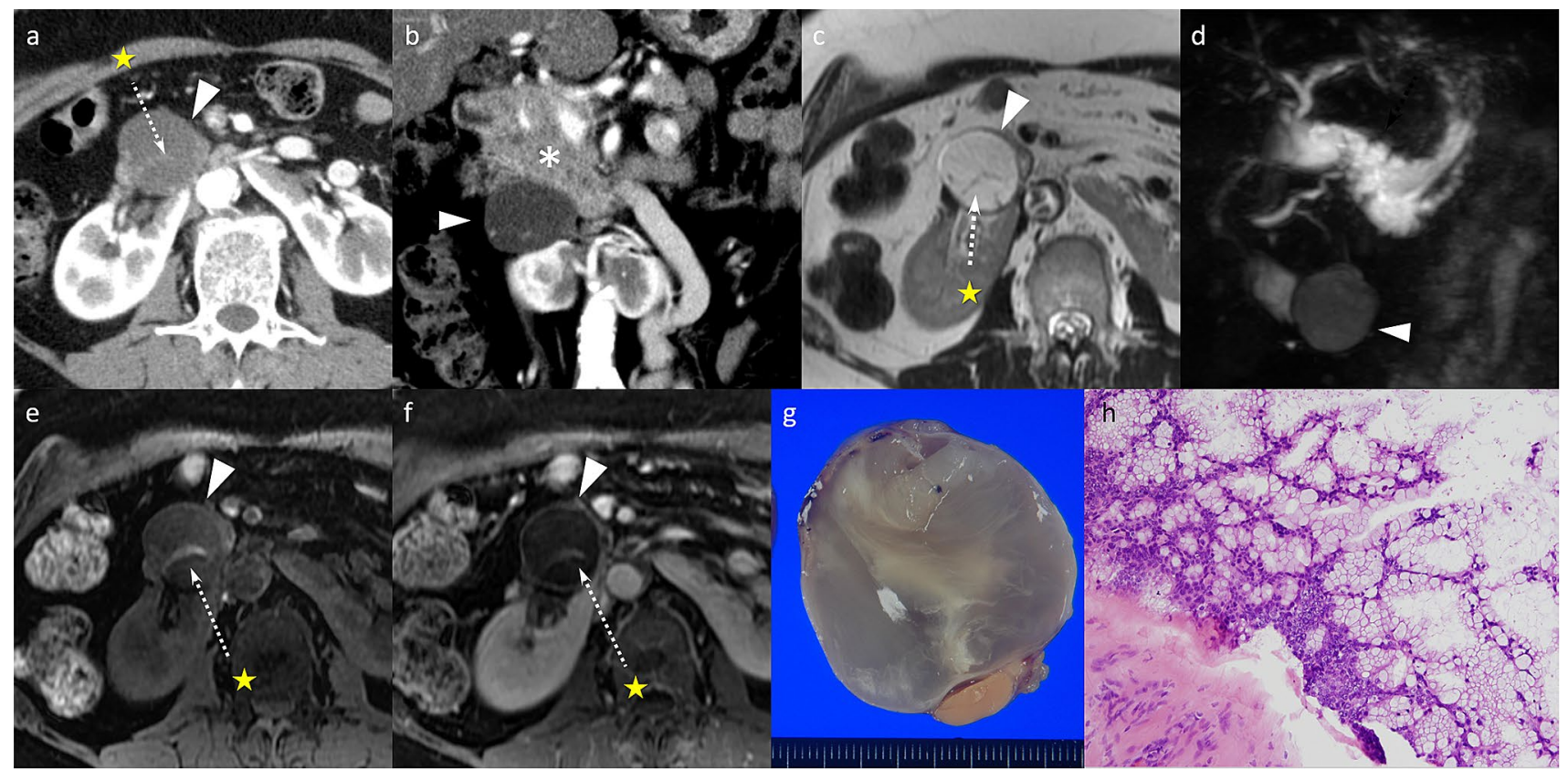

Fig. 20 Lesions in the peripancreatic area that mimic pancreatic cystic lesions. A 71-year-old woman with a cystic lesion in the pancreatic head mimicking MCN or lymphoepithelial cyst, which was diagnosed surgically as retroperitoneal mucinous cystic tumor. The irregular internal septa suggest $\mathrm{MCN}$, but the lesion was found to be extrapancreatic at surgery. It can be difficult to obtain an accurate diagnosis in lesions that are closely adjacent to the pancreatic parenchyma. a, b Arterial phase axial (a) and coronal (b) CT images reveal a cystic lesion (arrowhead) with internal septa (arrow) located adjacent to the head of the pancreas $(*)$. c The cystic lesion (arrowhead) shows clear hyperintensity and internal septa (arrow) on T2-weighted fat-suppressed MRI. d The lesion is depicted on MR cholangiopancreatography (arrowhead), but is slightly less distinct compared with a usual cystic lesion. e Precontrast T1-weighted fat-suppressed image shows a hypointense lesion (arrowhead) with internal septa (arrow). $\mathbf{f}$ Delayed phase image of dynamic contrasted MRI shows no enhancement of the mass (arrowhead) or internal septa (arrow). $\mathbf{g}$ The resected specimen shows a mucin-filled mass that was located in the retroperitoneum. $\mathbf{h}$ Histologically, the mass is lined by mucus-secreting columnar epithelial cells with mild nuclear atypia (HE staining; original magnification $\times 40$ ). The histological diagnosis is retroperitoneal mucinous cystic tumor
Table 1 Classification of pancreatic cystic lesions

\begin{tabular}{ll}
\hline Neoplastic cysts & Non-neoplastic cysts \\
Serous cystadenoma & Pseudocyst \\
Mucinous cystic neoplasm & Congenital cyst \\
Intraductal papillary mucinous neoplasm: branch duct, main & Retention cyst \\
duct, mixed & Hydatid cyst \\
Solid pseudopapillary neoplasm & Lymphoepithelial cyst \\
& Epidermoid cyst \\
Solid neoplasms with cystic degeneration & Para-pancreatic cystic lesions \\
Neuroendocrine tumor & Neurogenic tumor \\
Ductal adenocarcinoma & Metastatic lymph node \\
Acinar cell cystadenocarcinoma & Walled-off necrosis (after pancrea- \\
Metastatic tumor & titis) \\
& Others \\
\hline
\end{tabular}

Pancreatic cystic lesions are divided into neoplastic cysts, non-neoplastic cysts, solid neoplasm with cystic degeneration. Para-pancreatic cystic lesions should also be recognized. Neoplastic cysts other than serous cystadenomas have malignant potential

\section{Case presentations}

Most pancreatic cystic lesions show typical and well-known characteristic imaging features that easily indicate a specific diagnosis. In the setting of clinical practice, however, a definitive diagnosis is difficult in cases that show uncommon or overlapping imaging features. In the following pages, we present diagnostically challenging and instructive cases, along with radiologic-pathological correlations (Figs. 1, 2, $3,4,5,6,7,8,9,10,11,12,13,14,15,16,17,18,19,20)$. 
Table 2 Demographics and clinical features of pancreatic cystic lesions

\begin{tabular}{|c|c|c|c|c|c|c|c|c|c|}
\hline & $\begin{array}{l}\text { Serous cystad- } \\
\text { enoma }\end{array}$ & $\begin{array}{l}\text { Mucinous } \\
\text { cystic neo- } \\
\text { plasm }\end{array}$ & $\begin{array}{l}\text { Branch duct } \\
\text { IPMN }\end{array}$ & $\begin{array}{l}\text { Main duct } \\
\text { IPMN }\end{array}$ & Pseudocyst & $\begin{array}{l}\text { Solid pseu- } \\
\text { dopapillary } \\
\text { neoplasm }\end{array}$ & $\begin{array}{l}\text { Neuroendo- } \\
\text { crine tumor } \\
\text { with cystic } \\
\text { degeneration }\end{array}$ & $\begin{array}{l}\text { Lymphoepithe- } \\
\text { lial cyst }\end{array}$ & $\begin{array}{l}\text { Epidermoid } \\
\text { cyst }\end{array}$ \\
\hline Frequency & Moderate & Moderate & Common & Rare & Very common & Very rare & Common & Very rare & Very rare \\
\hline $\begin{array}{l}\text { Median age } \\
\text { (years) }\end{array}$ & $40-60$ & $40-50$ & $50-60$ & $50-60$ & Variably & $20-30$ & $50-60$ & $40-60$ & $40-60$ \\
\hline $\begin{array}{l}\text { Gender (male/ } \\
\text { female) }\end{array}$ & $\begin{array}{l}\text { Microcystic: } \\
\text { M>F (7:3); } \\
\text { Macrocystic: } \\
\text { M>F (3:2) }\end{array}$ & $\begin{array}{c}F>>>M \\
(20: 1)\end{array}$ & $\mathrm{M}>\mathrm{F}(2.2: 1)$ & $M>F(3: 2)$ & $\mathrm{M}>\mathrm{F}$ & $\mathrm{F}>>\mathrm{M}(9: 1)$ & $\mathrm{M}>\mathrm{F}$ & $\mathrm{M}>\mathrm{F}$ & $\mathrm{M} \approx \mathrm{F}$ \\
\hline $\begin{array}{l}\text { Symptoms } \\
\text { (e.g., pan- } \\
\text { creatitis) }\end{array}$ & Rare & $\begin{array}{l}\text { Only in large } \\
\text { tumors, } \\
\text { history of } \\
\text { pancreatitis } \\
\text { up to } 10 \%\end{array}$ & \multicolumn{2}{|c|}{$\begin{array}{l}\text { In up to one-third of cases mild } \\
\text { (recurrent) pancreatitis }\end{array}$} & $\begin{array}{l}\text { Often, history } \\
\text { of pan- } \\
\text { creatitis is } \\
\text { common }\end{array}$ & Rare & Usually none* & Usually none & Usually none \\
\hline
\end{tabular}

In addition to imaging characteristics of pancreatic cystic lesions, basic clinical factors are known and should be considered: frequency, age and gender of the patient, and symptoms. These can also help with an accurate diagnosis

$I P M N$ intraductal papillary mucinous neoplasm, $M$ male, $F$ female

*The presentation of functioning neuroendocrine tumors can be dramatic and depends on the hormones being produced

\section{Conclusion}

The many types of pancreatic cystic lesions present with various imaging appearances due to common and uncommon findings as well as overlapping imaging findings. Familiarity

Table 3 Key diagnostic imaging points with these whole entities and their characteristic appearances is helpful in making an accurate diagnosis.

\begin{tabular}{|c|c|}
\hline \multicolumn{2}{|l|}{ Characteristics of cystic lesions } \\
\hline $\begin{array}{l}\text { Morphological characteristics } \\
\text { Unilocular, microcystic, macrocystic, cyst } \\
\text { with solid component } \\
\text { Shape; lobulated, oval, grape-like } \\
\text { Cyst-in-cyst, cyst-on-cyst, cyst-by-cyst } \\
\text { Internal septa } \\
\text { Central scar }\end{array}$ & $\begin{array}{l}\text { Fluid content } \\
\text { Serous, mucinous } \\
\text { Containing sullage, hemorrhage, keratinized material, lipid } \\
\text { Necrotic material } \\
\text { Calcification } \\
\text { Frequency } \\
\text { Morphology; central, marginal (egg-shell-like), others }\end{array}$ \\
\hline $\begin{array}{l}\text { Wall } \\
\text { Thin, thick } \\
\text { Non-enhancing, enhancing } \\
\text { Homogeneous, irregular }\end{array}$ & $\begin{array}{l}\text { Localization } \\
\text { Head, body, tail } \\
\text { Exophytic or not } \\
\text { Number of lesions } \\
\text { Unifocal, multifocal }\end{array}$ \\
\hline $\begin{array}{l}\text { Mural nodules/solid components } \\
\text { Nodular, planular } \\
\text { Enhancing, non-enhancing }\end{array}$ & $\begin{array}{l}\text { Size, changes during observation } \\
\text { Original size } \\
\text { Enlargement, no change, shrinkage }\end{array}$ \\
\hline \multicolumn{2}{|c|}{ Pancreatic parenchymal change and peripancreatic findings } \\
\hline $\begin{array}{l}\text { Main pancreatic duct } \\
\text { Duct dilatation; diffuse, upstream or } \\
\text { downstream of the lesion } \\
\text { Communication with cyst }\end{array}$ & $\begin{array}{l}\text { Pancreatic parenchymal change } \\
\text { Parenchymal enlargement or atrophy; focal, diffuse } \\
\text { Changes in density or intensity because of edema } \\
\text { Parenchymal calcification } \\
\text { Peripancreatic findings } \\
\text { Fat stranding } \\
\text { Fluid collection } \\
\text { Walled-off necrosis }\end{array}$ \\
\hline
\end{tabular}




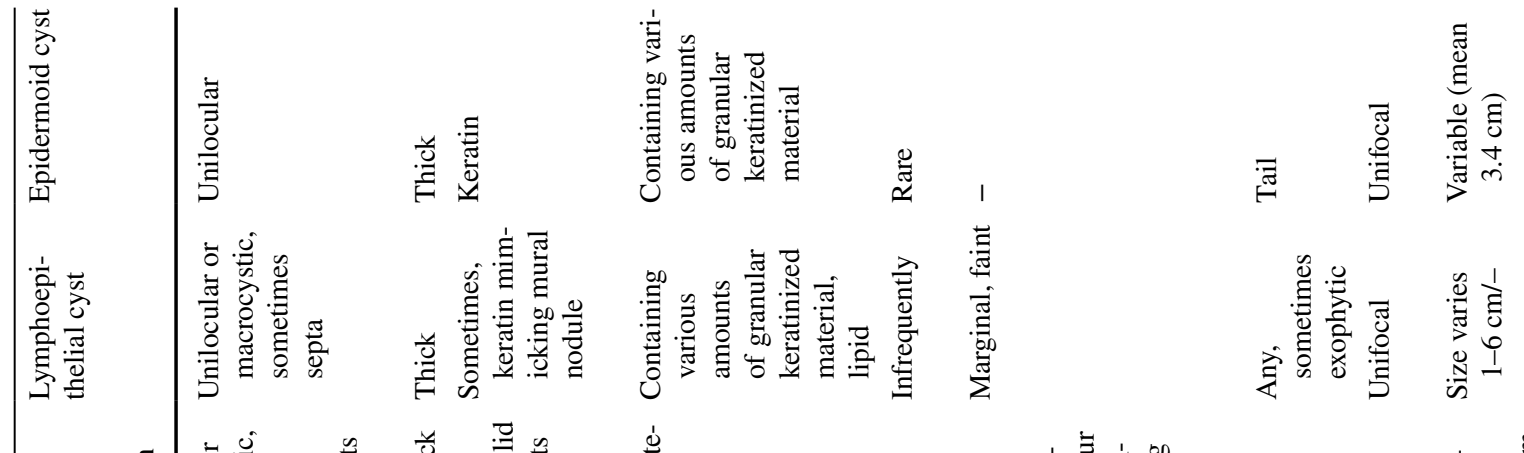

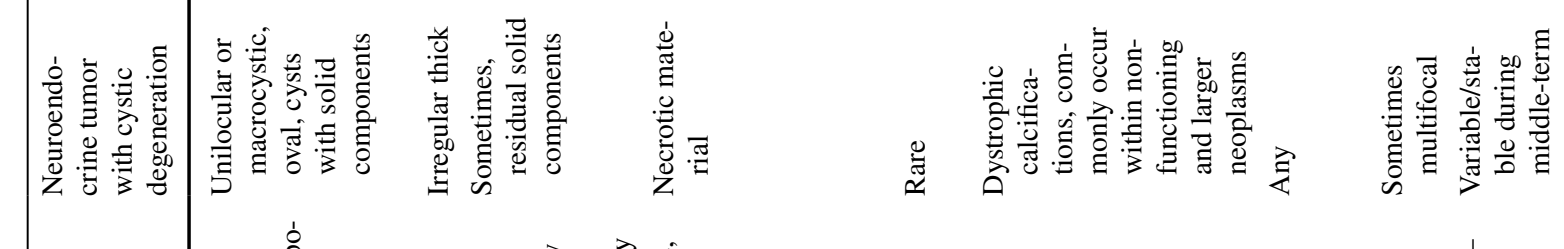

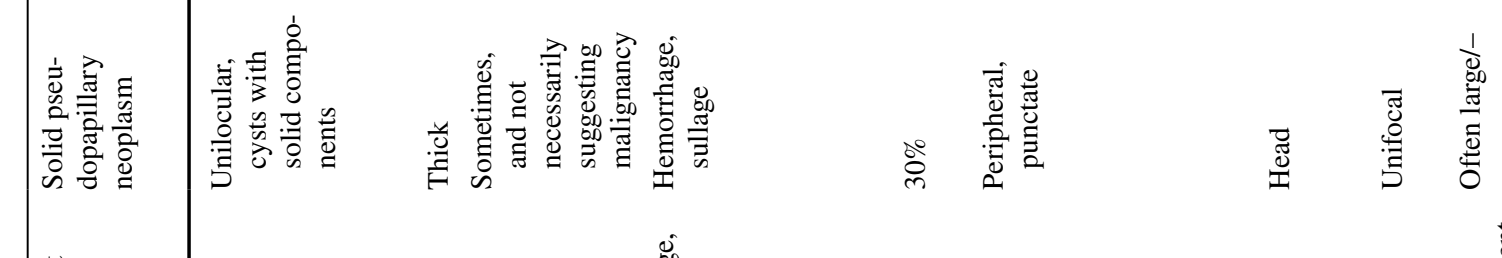

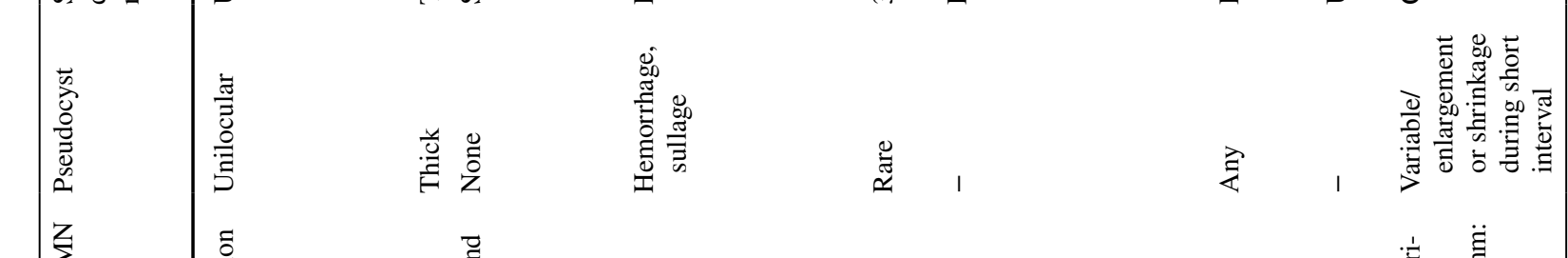

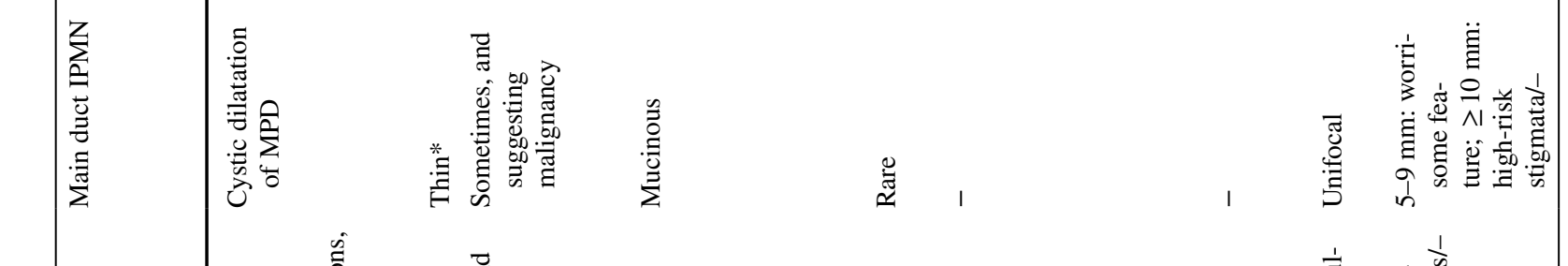

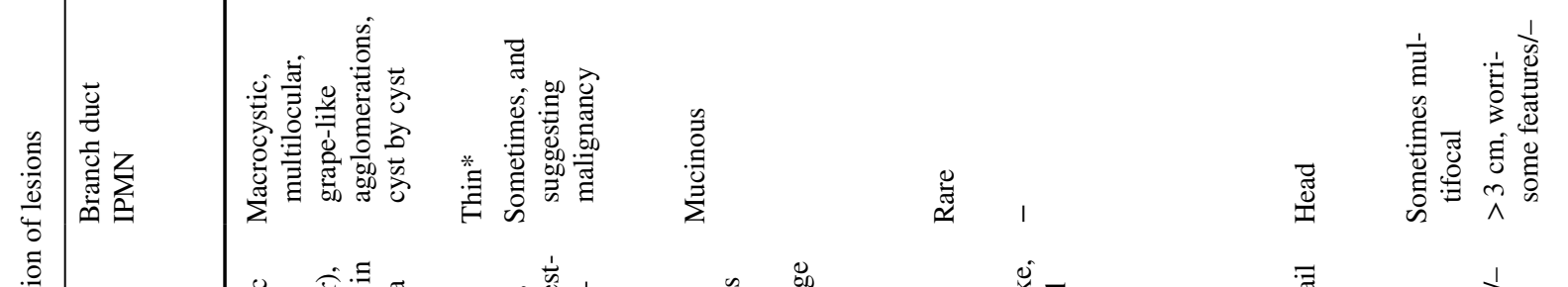

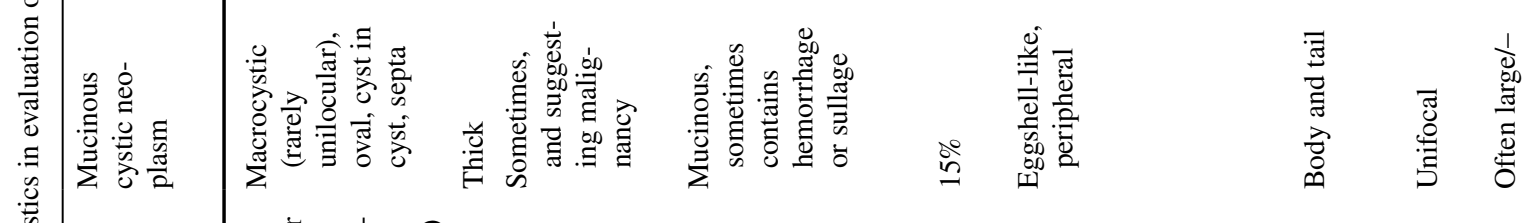

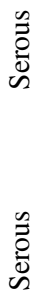

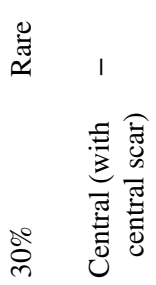

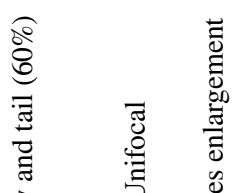

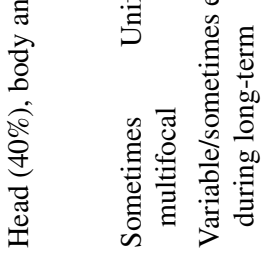

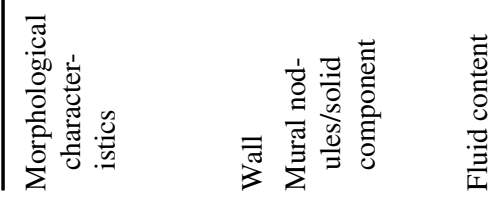

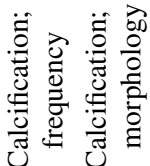

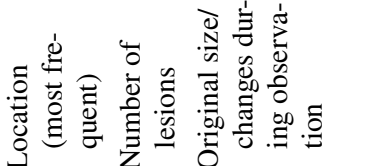




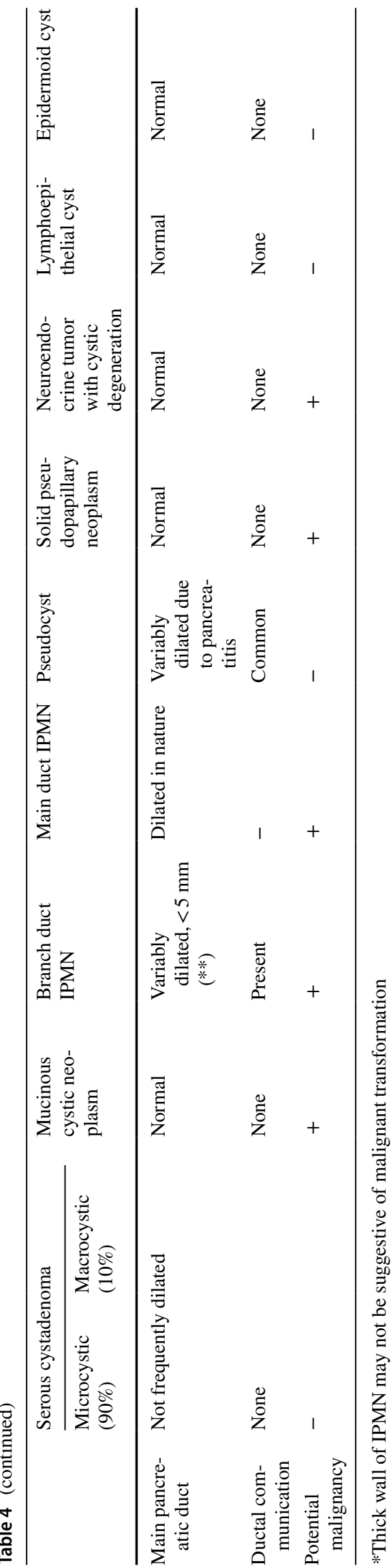

Funding This study received no specific grant from any funding agency.

\section{Compliance with ethical standards}

Conflict of interest The authors declare that they have no conflicts of interest.

Ethical approval All procedures performed in studies involving human participants were in accordance with the ethical standards of the institutional and/or national research committee and with the 1964 Helsinki Declaration and its later amendments or comparable ethical standards.

Informed consent The requirement for written informed consent was waived for this type of review article on the basis of the opt-out system of our institution

\section{References}

1. Spinelli KS, Fromwiller TE, Daniel RA, Kiely JM, Nakeeb A, Komorowski RA, et al. Cystic pancreatic neoplasms: observe or operate. Ann Surg. 2004;239:651-7 (discussion 57-9).

2. Laffan TA, Horton KM, Klein AP, Berlanstein B, Siegelman SS, Kawamoto S, et al. Prevalence of unsuspected pancreatic cysts on mdct. AJR Am J Roentgenol. 2008;191:802-7.

3. Lee HJ, Kim MJ, Choi JY, Hong HS, Kim KA. Relative accuracy of ct and mri in the differentiation of benign from malignant pancreatic cystic lesions. Clin Radiol. 2011;66:315-21.

4. Ferrone CR, Correa-Gallego C, Warshaw AL, Brugge WR, Forcione DG, Thayer SP, et al. Current trends in pancreatic cystic neoplasms. Arch Surg (Chicago, Ill: 1960). 2009;144:448-54.

5. Correa-Gallego C, Ferrone CR, Thayer SP, Wargo JA, Warshaw AL, Fernández-Del CC. Incidental pancreatic cysts: do we really know what we are watching? Pancreatology. 2010;10:144-50.

6. Javadi S, Menias CO, Korivi BR, Shaaban AM, Patnana M, Alhalabi K, et al. Pancreatic calcifications and calcified pancreatic masses: pattern recognition approach on ct. AJR Am J Roentgenol. 2017;209:77-87.

7. Kalb B, Sarmiento JM, Kooby DA, Adsay NV, Martin DR. $\mathrm{Mr}$ imaging of cystic lesions of the pancreas. Radiographics. 2009;29:1749-65.

8. Sahani DV, Kadavigere R, Saokar A, Fernandez-del CC, Brugge WR, Hahn PF. Cystic pancreatic lesions: a simple imaging-based classification system for guiding management. Radiographics. 2005;25:1471-84.

9. Sahani DV, Kambadakone A, Macari M, Takahashi N, Chari S, Fernandez-del CC. Diagnosis and management of cystic pancreatic lesions. AJR Am J Roentgenol. 2013;200:343-54.

10. Barral M, Soyer P, Dohan A, Laurent V, Hoeffel C, Fishman EK, et al. Magnetic resonance imaging of cystic pancreatic lesions in adults: An update in current diagnostic features and management. Abdom Imaging. 2014;39:48-65.

11. Palmucci S, Cappello G, Trombatore C, Tilocca C, Todaro R, Mauro LA, et al. Cystic pancreatic neoplasms: diagnosis and management emphasizing their imaging features. Eur Rev Med Pharm Sci. 2014;18:1259-68.

12. D’Onofrio M, De Robertis R, Capelli P, Tinazzi Martini P, Crosara $\mathrm{S}$, Gobbo $\mathrm{S}$, et al. Uncommon presentations of common pancreatic neoplasms: a pictorial essay. Abdom Imaging. 2015;40:1629-44.

13. Bollen TL, Wessels FJ. Radiological workup of cystic neoplasms of the pancreas. Visceral Med. 2018;34:182-90. 
14. Sahani DV, Lin DJ, Venkatesan AM, Sainani N, Mino-Kenudson $\mathrm{M}$, Brugge WR, et al. Multidisciplinary approach to diagnosis and management of intraductal papillary mucinous neoplasms of the pancreas. Clin Gastroenterol Hepatol. 2009;7:259-69.

15. Sakorafas GH, Smyrniotis V, Reid-Lombardo KM, Sarr MG. Primary pancreatic cystic neoplasms revisited. Part iii. Intraductal papillary mucinous neoplasms. Surg Oncol. 2011;20:e109-e118118.

16. Tanaka M, Fernández-del Castillo C, Adsay V, Chari S, Falconi M, Jang JY, et al. International consensus guidelines 2012 for the management of ipmn and mcn of the pancreas. Pancreatology. 2012;12:183-97.

17. Mino-Kenudson M, Fernández-del Castillo C, Baba Y, Valsangkar NP, Liss AS, Hsu M, et al. Prognosis of invasive intraductal papillary mucinous neoplasm depends on histological and precursor epithelial subtypes. Gut. 2011;60:1712-20.

18. Klöppel G. Pseudocysts and other non-neoplastic cysts of the pancreas. Semin Diagn Pathol. 2000;17:7-15.

19. Curry CA, Eng J, Horton KM, Urban B, Siegelman S, Kuszyk BS, et al. Ct of primary cystic pancreatic neoplasms: can ct be used for patient triage and treatment? AJR Am J Roentgenol. 2000;175:99-103.

20. Kosmahl M, Pauser U, Peters K, Sipos B, Lüttges J, Kremer B, et al. Cystic neoplasms of the pancreas and tumor-like lesions with cystic features: A review of 418 cases and a classification proposal. Virchows Arch. 2004;445:168-78

21. Fernández-del Castillo C, Warshaw AL. Cystic tumors of the pancreas. Surg Clin N Am. 1995;75:1001-166.

22. Sakorafas GH, Smyrniotis V, Reid-Lombardo KM, Sarr MG. Primary pancreatic cystic neoplasms of the pancreas revisited. Part iv: rare cystic neoplasms. Surg Oncol. 2012;21:153-63.

23. Lawrence B, Gustafsson BI, Chan A, Svejda B, Kidd M, Modlin IM. The epidemiology of gastroenteropancreatic neuroendocrine tumors. Endocrinol Metab Clin N Am. 2011;40(1-18):vii.

24. Lewis RB, Lattin GE Jr, Paal E. Pancreatic endocrine tumors: radiologic-clinicopathologic correlation. Radiographics. 2010;30:1445-644.

25. Hu S, Zhu L, Song Q, Chen K. Epidermoid cyst in intrapancreatic accessory spleen: computed tomography findings and clinical manifestation. Abdom Imaging. 2012;37:828-33.

26. Kanazawa H, Kamiya J, Nagino M, Uesaka K, Yuasa N, Oda K, et al. Epidermoid cyst in an intrapancreatic accessory spleen: a case report. J Hepato Biliary Pancreatic Surg. 2004;11:61-3.

27. Sakorafas GH, Smyrniotis V, Reid-Lombardo KM, Sarr MG. Primary pancreatic cystic neoplasms revisited: Part ii. Mucinous cystic neoplasms. Surg Oncol. 2011;20:e93-101.

28. Ventriglia A, Manfredi R, Mehrabi S, Boninsegna E, Negrelli R, Pedrinolla B, et al. Mri features of solid pseudopapillary neoplasm of the pancreas. Abdom Imaging. 2014;39:1213-20.

29. Sakorafas GH, Smyrniotis V, Reid-Lombardo KM, Sarr MG. Primary pancreatic cystic neoplasms revisited. Part i: serous cystic neoplasms. Surg Oncol. 2011;20:e84-92.

30. Levink I, Bruno MJ, Cahen DL. Management of intraductal papillary mucinous neoplasms: Controversies in guidelines and future perspectives. Curr Treat Opt Gastroenterol. 2018;16:316-32.

31. Nagai K, Doi R, Kida A, Kami K, Kawaguchi Y, Ito T, et al. Intraductal papillary mucinous neoplasms of the pancreas: clinicopathologic characteristics and long-term follow-up after resection. World J Surg. 2008;32:271-8 (discussion 79-80).

32. Schmidt CM, White PB, Waters JA, Yiannoutsos CT, Cummings OW, Baker M, et al. Intraductal papillary mucinous neoplasms: predictors of malignant and invasive pathology. Ann Surg. 2007;246:644-51 (discussion 51-4).
33. Ridtitid W, DeWitt JM, Schmidt CM, Roch A, Stuart JS, Sherman S, et al. Management of branch-duct intraductal papillary mucinous neoplasms: a large single-center study to assess predictors of malignancy and long-term outcomes. Gastrointest Endosc. 2016;84:436-45.

34. Marchegiani G, Andrianello S, Perri G, Pollini T, Caravati A, Secchettin E, et al. The role of age in pancreatic intraductal papillary mucinous neoplasms of the pancreas: same risk of death but different implications for management. Dig Liver Dis. 2018;50:1327-33.

35. Ilic M, Ilic I. Epidemiology of pancreatic cancer. World J Gastroenterol. 2016;22:9694-705.

36. Habashi S, Draganov PV. Pancreatic pseudocyst. World J Gastroenterol. 2009;15:38-47.

37. Crippa S, Salvia R, Warshaw AL, Domínguez I, Bassi C, Falconi M, et al. Mucinous cystic neoplasm of the pancreas is not an aggressive entity: lessons from 163 resected patients. Ann Surg. 2008:247:571-9.

38. Salvia R, Crippa S, Falconi M, Bassi C, Guarise A, Scarpa A, et al. Branch-duct intraductal papillary mucinous neoplasms of the pancreas: to operate or not to operate? Gut. 2007;56:1086-90.

39. Hata T, Sakata N, Okada T, Aoki T, Motoi F, Katayose Y, et al. Dilated papilla with mucin extrusion is a potential predictor of acute pancreatitis associated with intraductal papillary mucinous neoplasms of pancreas. Pancreatology. 2013;13:615-20.

40. Jang JW, Kim MH, Jeong SU, Kim J, Park DH, Lee SS, et al. Clinical characteristics of intraductal papillary mucinous neoplasm manifesting as acute pancreatitis or acute recurrent pancreatitis. $\mathbf{J}$ Gastroenterol Hepatol. 2013;28:731-8.

41. Pelletier AL, Hammel P, Rebours V, Couvelard A, Vullierme MP, Maire F, et al. Acute pancreatitis in patients operated on for intraductal papillary mucinous neoplasms of the pancreas: frequency, severity, and clinicopathologic correlations. Pancreas. 2010;39:658-61.

42. Ringold DA, Shroff P, Sikka SK, Ylagan L, Jonnalagadda S, Early DS, et al. Pancreatitis is frequent among patients with side-branch intraductal papillary mucinous neoplasia diagnosed by eus. Gastrointest Endosc. 2009;70:488-94.

43. Goh BK, Ooi LL, Tan YM, Cheow PC, Chung YF, Chow PK, et al. Clinico-pathological features of cystic pancreatic endocrine neoplasms and a comparison with their solid counterparts. Eur J Surg Oncol. 2006;32:553-6.

44. Ligneau B, Lombard-Bohas C, Partensky C, Valette PJ, Calender A, Dumortier J, et al. Cystic endocrine tumors of the pancreas: clinical, radiologic, and histopathologic features in 13 cases. Am J Surg Pathol. 2001;25:752-60.

45. Ro C, Chai W, Yu VE, Yu R. Pancreatic neuroendocrine tumors: biology, diagnosis, and treatment. Chin J Cancer. 2013;32:312-24.

46. Sheehan MK, Beck K, Pickleman J, Aranha GV. Spectrum of cystic neoplasms of the pancreas and their surgical management. Arch Surg Chicago, Ill: 1960). 2003;138:657-60 (discussion 60-2).

47. Fernández-del CC, Targarona J, Thayer SP, Rattner DW, Brugge WR, Warshaw AL. Incidental pancreatic cysts: clinicopathologic characteristics and comparison with symptomatic patients. Arch Surg Chicago, Ill: 1960). 2003;138:427-33 (discussion 33-4).

48. Brugge WR, Lauwers GY, Sahani D, Fernandez-del Castillo C, Warshaw AL. Cystic neoplasms of the pancreas. N Engl J Med. 2004;351:1218-26.

49. Choi JY, Kim MJ, Lee JY, Lim JS, Chung JJ, Kim KW, et al. Typical and atypical manifestations of serous cystadenoma of the pancreas: Imaging findings with pathologic correlation. AJR Am J Roentgenol. 2009;193:136-42. 
50. Sainani NI, Saokar A, Deshpande V, Fernández-del Castillo C, Hahn P, Sahani DV. Comparative performance of mdct and mri with $\mathrm{mr}$ cholangiopancreatography in characterizing small pancreatic cysts. AJR Am J Roentgenol. 2009;193:722-31.

51. Goh BK, Tan YM, Chung YF, Chow PK, Cheow PC, Wong WK, et al. A review of mucinous cystic neoplasms of the pancreas defined by ovarian-type stroma: clinicopathological features of 344 patients. World J Surg. 2006;30:2236-45.

52. Yao X, Ji Y, Zeng M, Rao S, Yang B. Solid pseudopapillary tumor of the pancreas: cross-sectional imaging and pathologic correlation. Pancreas. 2010;39:486-91.

53. Mirminachi B, Farrokhzad S, Sharifi AH, Nikfam S, Nikmanesh A, Malekzadeh R, et al. Solid pseudopapillary neoplasm of pancreas; a case series and review literature. Middle East J Dig Dis. 2016;8:102-8.

54. Kazumori H, Sizuku T, Ueki T, Uchida Y, Yamamoto S. Lymphoepithelial cyst of the pancreas. J Gastroenterol. 1997;32:700-3.

55. Adsay NV, Hasteh F, Cheng JD, Bejarano PA, Lauwers GY, Batts KP, et al. Lymphoepithelial cysts of the pancreas: a report of 12 cases and a review of the literature. Modern Pathol. 2002;15:492-501.

56. Rodriguez JR, Salvia R, Crippa S, Warshaw AL, Bassi C, Falconi $\mathrm{M}$, et al. Branch-duct intraductal papillary mucinous neoplasms: observations in 145 patients who underwent resection. Gastroenterology. 2007;133:72-9 (quiz 309-10).

57. Anand N, Sampath K, Wu BU. Cyst features and risk of malignancy in intraductal papillary mucinous neoplasms of the pancreas: a meta-analysis. Clin Gastroenterol Hepatol. 2013;11:91321 (quiz e59-60).

58. Kim KW, Park SH, Pyo J, Yoon SH, Byun JH, Lee MG, et al. Imaging features to distinguish malignant and benign branch-duct type intraductal papillary mucinous neoplasms of the pancreas: a meta-analysis. Ann Surg. 2014;259:72-81.

59. Kitano M, Sakamoto H, Komaki T, Kudo M. New techniques and future perspective of eus for the differential diagnosis of pancreatic malignancies: Contrast harmonic imaging. Dig Endosc. 2011;23(Suppl 1):46-50.

60. Manfredi R, Ventriglia A, Mantovani W, Mehrabi S, Boninsegna E, Zamboni G, et al. Mucinous cystic neoplasms and serous cystadenomas arising in the body-tail of the pancreas: Mr imaging characterization. Eur Radiol. 2015;25:940-9.

61. Lesniak RJ, Hohenwalter MD, Taylor AJ. Spectrum of causes of pancreatic calcifications. AJR Am J Roentgenol. 2002;178:79-86.

62. Loftus EV Jr, Olivares-Pakzad BA, Batts KP, Adkins MC, Stephens DH, Sarr MG, et al. Intraductal papillary-mucinous tumors of the pancreas: clinicopathologic features, outcome, and nomenclature. Members of the pancreas clinic, and pancreatic surgeons of mayo clinic. Gastroenterology. 1996;110:1909-18.

63. Buetow PC, Buck JL, Pantongrag-Brown L, Beck KG, Ros PR, Adair CF. Solid and papillary epithelial neoplasm of the pancreas: imaging-pathologic correlation on 56 cases. Radiology. 1996;199:707-11.

64. Kawamoto S, Lawler LP, Horton KM, Eng J, Hruban RH, Fishman EK. Mdct of intraductal papillary mucinous neoplasm of the pancreas: evaluation of features predictive of invasive carcinoma. AJR Am J Roentgenol. 2006;186:687-95.

65. Perez-Johnston R, Narin O, Mino-Kenudson M, Ingkakul T, Warshaw AL, Fernandez-Del Castillo C, et al. Frequency and significance of calcification in ipmn. Pancreatology. 2013;13:43-7.

66. Poultsides GA, Huang LC, Chen Y, Visser BC, Pai RK, Jeffrey $\mathrm{RB}$, et al. Pancreatic neuroendocrine tumors: radiographic calcifications correlate with grade and metastasis. Ann Surg Oncol. 2012;19:2295-303.

67. Jais B, Rebours V, Malleo G, Salvia R, Fontana M, Maggino L, et al. Serous cystic neoplasm of the pancreas: a multinational study of 2622 patients under the auspices of the international association of pancreatology and European pancreatic club (European study group on cystic tumors of the pancreas). Gut. 2016;65:305-12.

68. Salvia R, Partelli S, Crippa S, Landoni L, Capelli P, Manfredi $\mathrm{R}$, et al. Intraductal papillary mucinous neoplasms of the pancreas with multifocal involvement of branch ducts. Am J Surg. 2009;198:709-14.

69. Horton KM, Hruban RH, Yeo C, Fishman EK. Multi-detector row ct of pancreatic islet cell tumors. Radiographics. 2006;26:453-64.

70. Kawamoto S, Johnson PT, Shi C, Singhi AD, Hruban RH, Wolfgang CL, et al. Pancreatic neuroendocrine tumor with cyst like changes: evaluation with mdct. AJR Am J Roentgenol. 2013;200:W283-W290290.

71. Fritz S, Schirren M, Klauss M, Bergmann F, Hackert T, Hartwig W, et al. Clinicopathologic characteristics of patients with resected multifocal intraductal papillary mucinous neoplasm of the pancreas. Surgery. 2012;152:S74-80.

72. Sohn TA, Yeo CJ, Cameron JL, Hruban RH, Fukushima N, Campbell KA, et al. Intraductal papillary mucinous neoplasms of the pancreas: an updated experience. Ann Surg. 2004;239:788-97 (discussion 97-9).

73. Mohr VH, Vortmeyer AO, Zhuang Z, Libutti SK, Walther MM, Choyke PL, et al. Histopathology and molecular genetics of multiple cysts and microcystic (serous) adenomas of the pancreas in von hippel-lindau patients. Am J Pathol. 2000;157:1615-21.

74. Hammel PR, Vilgrain V, Terris B, Penfornis A, Sauvanet A, Correas JM, et al. Pancreatic involvement in von hippel-lindau disease. The groupe francophone d'etude de la maladie de von hippel-lindau. Gastroenterology. 2000;119:1087-95.

75. Kim JA, Blumenfeld JD, Chhabra S, Dutruel SP, Thimmappa ND, Bobb WO, et al. Pancreatic cysts in autosomal dominant polycystic kidney disease: prevalence and association with pkd2 gene mutations. Radiology. 2016;280:762-70.

76. Reddy RP, Smyrk TC, Zapiach M, Levy MJ, Pearson RK, Clain JE, et al. Pancreatic mucinous cystic neoplasm defined by ovarian stroma: Demographics, clinical features, and prevalence of cancer. Clin Gastroenterol Hepatol. 2004;2:1026-31.

77. Tseng JF, Warshaw AL, Sahani DV, Lauwers GY, Rattner DW, Fernandez-del CC. Serous cystadenoma of the pancreas: tumor growth rates and recommendations for treatment. Ann Surg. 2005;242:413-9 (discussion 19-21).

78. Freeny PC, Saunders MD. Moving beyond morphology: new insights into the characterization and management of cystic pancreatic lesions. Radiology. 2014;272:345-63.

79. Allen PJ, D’Angelica M, Gonen M, Jaques DP, Coit DG, Jarnagin WR, et al. A selective approach to the resection of cystic lesions of the pancreas: results from 539 consecutive patients. Ann Surg. 2006;244:572-82.

80. Buscaglia JM, Giday SA, Kantsevoy SV, Jagannath SB, Magno P, Wolfgang CL, et al. Patient- and cyst-related factors for improved prediction of malignancy within cystic lesions of the pancreas. Pancreatology. 2009;9:631-8.

81. Di Benedetto F, Ballarin R, De Ruvo N, Berretta M, Spaggiari M, Montalti R, et al. Pancreatic resections for malignancy in patients aged 70 and older. J Am Geriatr Soc. 2009;57:1323-4.

82. Pitman MB, Lewandrowski K, Shen J, Sahani D, Brugge W, Fernandez-del CC. Pancreatic cysts: preoperative diagnosis and clinical management. Cancer Cytopathol. 2010;118:1-13.

83. Kim SY, Lee JM, Kim SH, Shin KS, Kim YJ, An SK, et al. Macrocystic neoplasms of the pancreas: $\mathrm{Ct}$ differentiation of serous oligocystic adenoma from mucinous cystadenoma and intraductal papillary mucinous tumor. AJR Am J Roentgenol. 2006;187:1192-8.

84. Stamm BH. Incidence and diagnostic significance of minor pathologic changes in the adult pancreas at autopsy: a systematic study 
of 112 autopsies in patients without known pancreatic disease. Hum Pathol. 1984;15:677-83.

85. Sahani DV, Kadavigere R, Blake M, Fernandez-Del Castillo C, Lauwers GY, Hahn PF. Intraductal papillary mucinous neoplasm of pancreas: multi-detector row ct with $2 \mathrm{~d}$ curved reformationscorrelation with mrcp. Radiology. 2006;238:560-9.

86. Singhal D, Kakodkar R, Sud R, Chaudhary A. Issues in management of pancreatic pseudocysts. JOP J Pancreas. 2006;7:502-7.

87. Rosenberger LH, Stein LH, Witkiewicz AK, Kennedy EP, Yeo CJ. Intraductal papillary mucinous neoplasm (ipmn) with extra-pancreatic mucin: a case series and review of the literature. J Gastrointest Surg. 2012;16:762-70.

Publisher's Note Springer Nature remains neutral with regard to jurisdictional claims in published maps and institutional affiliations. 